\title{
Relaxed formulation of the design conditions for Takagi-Sugeno fuzzy virtual actuators
}

\author{
ANNA FILASOVÁ, DUŠAN KROKAVEC and PAVOL LIŠČINSKÝ
}

\begin{abstract}
The $\mathrm{H}_{\infty}$ norm approach to virtual actuators design, intended to Takagi-Sugeno fuzzy continuous-time systems, is presented in the paper. Using the second Ljapunov method, the design conditions are formulated in terms of linear matrix inequalities in adapted bounded real lemma structures. Related to the static output controller, and for systems under influence of single actuator faults, the design steps are revealed for a three-tank system plant.
\end{abstract}

Key words: nonlinear dynamic systems, Takagi-Sugeno fuzzy models, fault tolerant control, static output controllers, virtual actuators, linear matrix inequalities.

\section{Introduction}

To increase the reliability of systems, fault tolerant control (FTC) usually fix a system with faults for continuing its mission, while different approaches were studied in FTC design (see, e.g., [1], [3], [15], [21], [23] and the references therein). The standard approach to control reconfiguration discards the nominal controller from the control loop and replace it with a new one with re-tuned parameters, to recover in a certain extent the performance of the fault-free control system [14], [17], [24]. By contrast, instead of adapting the controller to the faulty plant, the virtual approach keeps the nominal controller in the reconfigured closed-loop system and virtually adapt the faulty plant to the nominal controller [2], [13]. The reconfiguration block is chosen so as to hide a fault for the controller input and offers a way for the minimum invasive control reconfiguration [17], [18]. Designated to sensor faults the reconfiguration block is termed virtual sensor, while in actuator faults is named virtual actuator.

Considering the properties of Takagi-Sugeno (TS) fuzzy models for a class of nonlinear systems [4], [19], [20], [22], the approach proposed in the paper adapts the virtual

The authors are with Technical University of Košice, Faculty of Electrical Engineering and Informatics, Department of Cybernetics and Artificial Intelligence, Letná 9, 04200 Košice, Slovakia, fax: +421 55625 3574, e-mails: anna.filasova@tuke.sk, dusan.krokavec@tuke.sk, pavol.liscinsky@tuke.sk

The work presented in this paper was supported by VEGA, the Grant Agency of the Ministry of Education and the Academy of Science of Slovak Republic, under Grant No. 1/0348/14. This support is very gratefully acknowledged.

Received 18.12.2015. 
actuator technique for TS fuzzy systems. To achieve the desired control objectives, the design conditions for TS fuzzy static output controllers as well as for TS fuzzy virtual actuators are formulated using linear matrix inequality (LMI) techniques, by exploiting the bounded real lemma approach, and by adapting the generalized $\mathrm{H}_{\infty}$ norm principle presented in [10], [11] to the standard Lyapunov second method.

The paper is organized as follows. Continuing with system description in Sec. 2 the methods for TS fuzzy static output controller design, exploiting $\mathrm{H}_{\infty}$ approach, are given in Sec. 3 Demonstrating the separation principle for TS fuzzy virtual actuator design in Sec. 4 the desired specifications, as well as the LMI forms of the design conditions for TS fuzzy virtual actuator, are proven in Sec. 5 In response, Sec. 6 shows the performance of the proposed approach using an application example and Sec. 7 gives some concluding remarks.

Throughout the paper, the following notations are used: $\boldsymbol{x}^{T}, \boldsymbol{X}^{T}$ denotes the transpose of the vector $\boldsymbol{x}$ and the matrix $\boldsymbol{X}$, respectively, for a square matrix $\boldsymbol{X}<0$ means that $\boldsymbol{X}$ is symmetric negative definite matrix, $\operatorname{rank}(\cdot)$ remits the rank of a matrix, $\operatorname{diag}[\cdot]$ designates a block diagonal matrix, the symbol $\boldsymbol{I}_{n}$ indicates the $n$-th order unit matrix, $\mathbb{R}$ identifies the set of real numbers and $\mathbb{R}^{n}, \mathbb{R}^{n \times r}$ refers to the set of all $n$-dimensional real vectors and $n \times r$ real matrices, respectively.

\section{System descriptions}

The considered class of the Takagi-Sugeno continuous-time dynamic systems is described in the fault-free conditions as

$$
\begin{gathered}
\dot{\boldsymbol{q}}(t)=\sum_{i=1}^{s} \mathrm{~h}_{i}(\boldsymbol{\theta}(t))\left(\boldsymbol{A}_{i} \boldsymbol{q}(t)+\boldsymbol{B}_{i} \boldsymbol{u}_{c}(t)+\boldsymbol{V}_{i} \boldsymbol{d}(t)\right), \\
\boldsymbol{y}(t)=\boldsymbol{C} \boldsymbol{q}(t),
\end{gathered}
$$

where $\boldsymbol{q}(t) \in \mathbb{R}^{n}, \boldsymbol{u}_{c}(t) \in \mathbb{R}^{r}, \boldsymbol{y}(t) \in \mathbb{R}^{m}$ stand for state, control input and measurable output, $\boldsymbol{d}(t) \in \mathbb{R}^{p}$ is unknown disturbance, the system matrices $\boldsymbol{A}_{i} \in \mathbb{R}^{n \times n}, \boldsymbol{B}_{i} \in \mathbb{R}^{n \times r}$, $\boldsymbol{V}_{i} \in \mathbb{R}^{n \times r_{v}}, \boldsymbol{C} \in \mathbb{R}^{m \times n}$ for all $i$ are finite valued and $m=r$.

The variables $\theta_{j}(t), j=1,2, \ldots, o$, tied with the sector TS fuzzy model, span the $o$-dimensional vector of premise variables

$$
\boldsymbol{\theta}(t)=\left[\begin{array}{llll}
\theta_{1}(t) & \theta_{2}(t) & \cdots & \theta_{o}(t)
\end{array}\right] .
$$

It is supposed that the measurable premise variables, the nonlinear sectors and the normalized membership functions $\mathrm{h}_{i}(\boldsymbol{\theta}(t))$ are chosen in such a way that the pairs $\left(\boldsymbol{A}_{i}, \boldsymbol{B}_{i}\right)$ are stabilizable and the pairs $\left(\boldsymbol{A}_{i}, \boldsymbol{C}\right)$ are detectable for for all $i$ [5], while none premise variable is independent of the elements of the input vector $\boldsymbol{u}(t)$. More details can be found, e.g., in [4], [8], [9]. 
The state-space description of the system with a single actuator fault is considered as follows

$$
\begin{gathered}
\dot{\boldsymbol{q}}_{f a}(t)=\sum_{i=1}^{s} \mathrm{~h}_{i}(\boldsymbol{\theta}(t))\left(\boldsymbol{A}_{i} \boldsymbol{q}_{f a}(t)+\boldsymbol{B}_{f i} \boldsymbol{u}_{f a}(t)+\boldsymbol{V}_{i} \boldsymbol{d}(t)\right), \\
\boldsymbol{y}_{f a}(t)=\boldsymbol{C} \boldsymbol{q}_{f a}(t),
\end{gathered}
$$

where $\boldsymbol{q}_{f a}(t) \in \mathbb{R}^{n}$ denotes the system state variables vector, $\boldsymbol{u}_{f a}(t) \in \mathbb{R}^{r_{f}}$ labels the vector of acting control input variables, $\boldsymbol{y}_{f a}(t) \in \mathbb{R}^{m}$ identifies the vector of output variables, while the matrices $\boldsymbol{B}_{f i} \in \mathbb{R}^{n \times r_{f}}$ are of finite valued for all $i$ and $\operatorname{rank}\left(\boldsymbol{B}_{f i}\right)<\operatorname{rank}\left(\boldsymbol{B}_{i}\right)$. Moreover, it is supposed that the pairs $\left(\boldsymbol{A}_{i}, \boldsymbol{B}_{f i}\right)$ are controllable for all $i$ and the input vector $\boldsymbol{u}_{f a}(t)$ is available for reconfiguration (all inputs to the plant are available as they use the nominal controller, but one associated with the faulty actuator is broken). Moreover, it is believed that the nonlinear sectors boundaries are the same for the nominal or the faulty TS fuzzy model.

\section{Nominal TS fuzzy static output controller}

Using the same set of membership functions, the fuzzy static output controller in the parallel distributed form is defined as [8]

$$
\boldsymbol{u}_{c}(t)=-\sum_{j=1}^{s} \mathrm{~h}_{j}(\boldsymbol{\theta}(t)) \boldsymbol{K}_{j} \boldsymbol{y}(t)=-\sum_{j=1}^{s} \mathrm{~h}_{j}(\boldsymbol{\theta}(t)) \boldsymbol{K}_{j} \boldsymbol{C} \boldsymbol{q}(t),
$$

where $\left\{\boldsymbol{K}_{j} \in \mathbb{R}^{r \times m}, j=1,2, \ldots, s\right\}$ is the set of control gain matrices. Therefore, the nominal closed-loop system with unknown disturbance is described as

$$
\begin{gathered}
\dot{\boldsymbol{q}}(t)=\sum_{i=1}^{s} \sum_{j=1}^{s} \mathrm{~h}_{i}(\boldsymbol{\theta}(t)) \mathrm{h}_{j}(\boldsymbol{\theta}(t))\left(\boldsymbol{A}_{c i j} \boldsymbol{q}(t)+\boldsymbol{V}_{i} \boldsymbol{d}(t)\right), \\
\boldsymbol{y}(t)=\boldsymbol{C} \boldsymbol{q}(t),
\end{gathered}
$$

where

$$
\boldsymbol{A}_{c i j}=\boldsymbol{A}_{i}-\boldsymbol{B}_{i} \boldsymbol{K}_{j} \boldsymbol{C}, i, j=1,2, \ldots s .
$$

The design conditions are given by the following theorem.

Theorem 6 The equilibrium of the fuzzy system (1), (2), controlled by the fuzzy controller (6), is globally asymptotically stable with quadratic constraint $\gamma$ if there exist a positive definite symmetric matrix $\boldsymbol{R} \in \mathbb{R}^{n \times n}$, matrices $\boldsymbol{M} \in \mathbb{R}^{m \times m}, \boldsymbol{N}_{j} \in \mathbb{R}^{r \times n}$, $\boldsymbol{Y}_{i j} \in \mathbb{R}^{n \times n}$ and a positive scalar $\boldsymbol{\gamma} \in \mathbb{R}$ such that for all $i \in\langle 1,2, \ldots s\rangle, i<j \leqslant s$, 
$i, j \in\langle 1,2, \ldots s\rangle$, respectively, and $h_{i}(\boldsymbol{\theta}(t)) h_{j}(\boldsymbol{\theta}(t)) \neq 0$

$$
\begin{aligned}
& \boldsymbol{Y}^{\bullet}=\left[\begin{array}{cccc}
\boldsymbol{Y}_{11} & \boldsymbol{Y}_{12} & \cdots & \boldsymbol{Y}_{1 s} \\
\boldsymbol{Y}_{21} & \boldsymbol{Y}_{22} & \cdots & \boldsymbol{Y}_{2 s} \\
\vdots & \vdots & \ddots & \vdots \\
\boldsymbol{Y}_{s 1} & \boldsymbol{Y}_{s 2} & \cdots & \boldsymbol{Y}_{s s}
\end{array}\right]>0, \quad \boldsymbol{Y}_{i j}=\boldsymbol{Y}_{j i}^{T}, \\
& \boldsymbol{R}=\boldsymbol{R}^{T}>0, \\
& M C=C R, \\
& {\left[\begin{array}{ccc}
\boldsymbol{H}_{i i}+\boldsymbol{H}_{i i}^{T}+\boldsymbol{Y}_{i i} & * & * \\
\boldsymbol{V}_{i}^{T} & -\gamma \boldsymbol{I}_{r} & * \\
\boldsymbol{C R} & \mathbf{0} & -\boldsymbol{I}_{m}
\end{array}\right]<0,} \\
& {\left[\begin{array}{ccc}
\frac{\boldsymbol{H}_{i j}+\boldsymbol{H}_{j i}}{2}+\frac{\boldsymbol{H}_{i j}^{T}+\boldsymbol{H}_{j i}^{T}}{2}+\frac{\boldsymbol{Y}_{i j}+\boldsymbol{Y}_{j i}}{2} & * & * \\
\frac{\boldsymbol{V}_{i}^{T}+\boldsymbol{V}_{j}^{T}}{2} & -\gamma \boldsymbol{I}_{r} & * \\
\boldsymbol{C} \boldsymbol{R} & \mathbf{0} & -\boldsymbol{I}_{m}
\end{array}\right]<0,}
\end{aligned}
$$

where

$$
\boldsymbol{H}_{i j}=A_{i} \boldsymbol{R}-\boldsymbol{B}_{i} \boldsymbol{N}_{j} \boldsymbol{C}
$$

and $\boldsymbol{I}_{r} \in \mathbb{R}^{r \times r}, \boldsymbol{I}_{m} \in \mathbb{R}^{m \times m}$ are identity matrices.

If the above conditions hold, the set of control law gain matrices is given as

$$
\boldsymbol{K}_{j}=\boldsymbol{N}_{j} \boldsymbol{M}^{-1}, \quad j=1,2, \ldots, s
$$

Hereafter, $*$ denotes the symmetric item in a symmetric matrix.

Proof (compare [8]) Considering the quadratic Lyapunov function of the form

$$
v(\boldsymbol{q}(t))=\boldsymbol{q}^{T}(t) \boldsymbol{P} \boldsymbol{q}(t)+\int_{0}^{t}\left(\boldsymbol{y}^{T}(\tau) \boldsymbol{y}(\tau)-\gamma \boldsymbol{d}^{T}(\tau) \boldsymbol{d}(\tau)\right) \mathrm{d} \tau>0
$$

where $\boldsymbol{P} \in \mathbb{R}^{n \times n}$ is a positive definite symmetric matrix and $\gamma \in \mathbb{R}$ is square of the $\mathrm{H}_{\infty}$ norm of the disturbance transfer function matrix, then the time derivative of $v(\boldsymbol{q}(t))$ is

$$
\dot{v}(\boldsymbol{q}(t))=\dot{\boldsymbol{q}}^{T}(t) \boldsymbol{P} \boldsymbol{q}(t)+\boldsymbol{q}^{T}(t) \boldsymbol{P} \dot{\boldsymbol{q}}(t)+\boldsymbol{y}^{T}(t) \boldsymbol{y}(t)-\gamma \boldsymbol{d}^{T}(t) \boldsymbol{d}(t)<0 .
$$

Substituting (7), (8) into (18), and introducing the quadratic term

$$
v_{v}(\boldsymbol{\theta}(t))=\boldsymbol{q}^{T}(t) \mathbf{Z}(\boldsymbol{\theta}(t)) \boldsymbol{q}(t),
$$




$$
\boldsymbol{Z}(\boldsymbol{\theta}(t))=\sum_{i=1}^{s} \sum_{j=1}^{s} \mathrm{~h}_{i}(\boldsymbol{\theta}(t)) \mathrm{h}_{j}(\boldsymbol{\theta}(t)) \boldsymbol{X}_{i j}>0
$$

while $\left\{\boldsymbol{X}_{i j}=\boldsymbol{X}_{i j}^{T} \in \mathbb{R}^{n \times n}, i, j=1,2, \ldots, s\right\}$ is the set of matrices, then in the sense of Krasovskii theorem (see, e.g., [7]) it can be set up

$$
\begin{gathered}
\dot{v}(\boldsymbol{q}(t))= \\
=\sum_{i=1}^{s} \sum_{j=1}^{s} \mathrm{~h}_{i}(\boldsymbol{\theta}(t)) \mathrm{h}_{j}(\boldsymbol{\theta}(t)) \boldsymbol{q}^{T}(t)\left(\boldsymbol{P} \boldsymbol{A}_{c i j}+\boldsymbol{A}_{c i j}^{T} \boldsymbol{P}\right) \boldsymbol{q}(t)+ \\
+\boldsymbol{q}^{T}(t) \boldsymbol{P} \boldsymbol{V}_{i} \boldsymbol{d}(t)+\boldsymbol{d}^{T}(t) \boldsymbol{V}_{i}^{T} \boldsymbol{P} \boldsymbol{d}(t)+\boldsymbol{q}^{T}(t) \boldsymbol{C}^{T} \boldsymbol{C} \boldsymbol{q}(t)-\gamma \boldsymbol{d}^{T}(t) \boldsymbol{d}(t) \leqslant \\
\leqslant-\sum_{i=1}^{s} \sum_{j=1}^{s} \mathrm{~h}_{i}(\boldsymbol{\theta}(t)) \mathrm{h}_{j}(\boldsymbol{\theta}(t)) \boldsymbol{q}^{T}(t) \boldsymbol{X}_{i j} \boldsymbol{q}(t)<0 .
\end{gathered}
$$

Using the notation

$$
\boldsymbol{q}_{c}^{T}(t)=\left[\begin{array}{ll}
\boldsymbol{q}^{T}(t) & \boldsymbol{d}^{T}(t)
\end{array}\right]
$$

(21) can be written as

$$
\dot{v}\left(\boldsymbol{q}_{c}(t)\right)=\sum_{i=1}^{s} \sum_{j=1}^{s} \mathrm{~h}_{i}(\boldsymbol{\theta}(t)) \mathrm{h}_{j}(\boldsymbol{\theta}(t)) \boldsymbol{q}^{T}(t) \boldsymbol{P}_{c i j} \boldsymbol{q}(t)<0,
$$

where

$$
\boldsymbol{P}_{c i j}=\left[\begin{array}{cc}
\boldsymbol{P A}_{c i j}+\boldsymbol{A}_{c i j}^{T} \boldsymbol{P}+\boldsymbol{X}_{i j}+\boldsymbol{C}^{T} \boldsymbol{C} & \boldsymbol{P} \boldsymbol{V}_{i} \\
\boldsymbol{V}_{i}^{T} \boldsymbol{P} & -\gamma \boldsymbol{I}_{r}
\end{array}\right]<0 .
$$

Permuting the subscripts $i$ and $j$ in (23) gives

$$
\dot{v}\left(\boldsymbol{q}_{c}(t)\right)=\sum_{i=1}^{s} \sum_{j=1}^{s} \mathrm{~h}_{i}(\boldsymbol{\theta}(t)) \mathrm{h}_{j}(\boldsymbol{\theta}(t)) \boldsymbol{q}^{T}(t) \boldsymbol{P}_{c j i} \boldsymbol{q}(t)<0
$$

and adding of (23) and (25) results in

$$
2 \dot{v}\left(\boldsymbol{q}_{c}(t)\right)=\sum_{i=1}^{s} \sum_{j=1}^{s} \mathrm{~h}_{i}(\boldsymbol{\theta}(t)) \mathrm{h}_{j}(\boldsymbol{\theta}(t)) \boldsymbol{q}^{T}(t)\left(\boldsymbol{P}_{c i j}+\boldsymbol{P}_{c j i}\right) \boldsymbol{q}(t)<0 .
$$

Rearranging the computation, (26) takes the form

$$
\begin{gathered}
\dot{v}(\boldsymbol{q}(t))= \\
=\sum_{i=1}^{s} \mathrm{~h}_{i}^{2}(\boldsymbol{\theta}(t)) \boldsymbol{q}^{T}(t) \boldsymbol{P}_{c i i} \boldsymbol{q}(t)+2 \sum_{i=1}^{s-1} \sum_{j=i+1}^{s} \mathrm{~h}_{i}(\boldsymbol{\theta}(t)) \mathrm{h}_{j}(\boldsymbol{\theta}(t)) \boldsymbol{q}^{T}(t) \frac{\boldsymbol{P}_{c i j}+\boldsymbol{P}_{c j i}}{2} \boldsymbol{q}(t)<0 .
\end{gathered}
$$

Thus, for all $i \in\langle 1,2, \ldots s\rangle, i<j \leqslant s, i, j \in\langle 1,2, \ldots s\rangle$, respectively, (27) implies

$$
\boldsymbol{P}_{c i i}<0, \quad \frac{\boldsymbol{P}_{c i j}+\boldsymbol{P}_{c j i}}{2}<0 .
$$


Pre-multiplying the left-hand side and post-multiplying the right-hand of the matrices $\boldsymbol{P}_{c i i}, \boldsymbol{P}_{c j i}$ by the transform matrix

$$
\boldsymbol{S}=\operatorname{diag}\left[\begin{array}{ll}
\boldsymbol{R} & \boldsymbol{I}_{r}
\end{array}\right], \quad \boldsymbol{R}=\boldsymbol{P}^{-1}
$$

the bilinear matrix inequalities (28) can be partly linearized. Thus, as a consequence, (24) and (28) give

$$
\begin{gathered}
{\left[\begin{array}{cc}
\boldsymbol{A}_{c i i} \boldsymbol{R}+\boldsymbol{R} \boldsymbol{A}_{c i i}^{T}+\boldsymbol{R} \boldsymbol{X}_{i i} \boldsymbol{R}+\boldsymbol{R} \boldsymbol{C}^{T} \boldsymbol{C} \boldsymbol{R} & \boldsymbol{V}_{i} \\
\boldsymbol{V}_{i}^{T} & -\boldsymbol{\gamma} \boldsymbol{I}_{r}
\end{array}\right]<0,} \\
{\left[\begin{array}{cc}
\frac{\boldsymbol{A}_{c i j}+\boldsymbol{A}_{c j i}}{2} \boldsymbol{R}+\boldsymbol{R} \frac{\boldsymbol{A}_{c i j}^{T}+\boldsymbol{A}_{c j i}^{T}}{2}+\boldsymbol{R}_{i j}^{\frac{\boldsymbol{X}_{i j}+\boldsymbol{R}_{j i}}{2}} \boldsymbol{R}+\boldsymbol{R} \boldsymbol{C}^{T} \boldsymbol{C} \boldsymbol{R} & \frac{\boldsymbol{V}_{i}+\boldsymbol{V}_{j}}{2} \\
\frac{\boldsymbol{V}_{i}^{T}+\boldsymbol{V}_{j}^{T}}{2} & -\gamma \boldsymbol{I}_{r}
\end{array}\right]<0,}
\end{gathered}
$$

respectively, and by using the Schur complement property then (30), (31) imply

$$
\begin{aligned}
& {\left[\begin{array}{ccc}
\boldsymbol{A}_{c i i} \boldsymbol{R}+\boldsymbol{R} \boldsymbol{A}_{c i i}^{T}+\boldsymbol{R} \boldsymbol{X}_{i i} \boldsymbol{R} & \boldsymbol{V}_{i} & \boldsymbol{R} \boldsymbol{C}^{T} \\
\boldsymbol{V}_{i}^{T} & -\boldsymbol{\gamma} \boldsymbol{I}_{r} & \mathbf{0} \\
\boldsymbol{C} \boldsymbol{R} & \mathbf{0} & -\boldsymbol{I}_{m}
\end{array}\right]<0,} \\
& {\left[\begin{array}{ccc}
\frac{\boldsymbol{A}_{c i j}+\boldsymbol{A}_{c j i}}{2} \boldsymbol{R}+\boldsymbol{R} \frac{\boldsymbol{A}_{c i j}^{T}+\boldsymbol{A}_{c j i}^{T}}{2}+\boldsymbol{R} \frac{\boldsymbol{X}_{i j}+\boldsymbol{X}_{j i}}{2} \boldsymbol{R} & \frac{\boldsymbol{V}_{i}+\boldsymbol{V}_{j}}{2} & \boldsymbol{R} \boldsymbol{C}^{T} \\
\frac{\boldsymbol{V}_{i}^{T}+\boldsymbol{V}_{j}^{T}}{2} & -\boldsymbol{\gamma} \boldsymbol{I}_{r} & \mathbf{0} \\
\boldsymbol{C} \boldsymbol{R} & \mathbf{0} & -\boldsymbol{I}_{m}
\end{array}\right]<0 .}
\end{aligned}
$$

Writing as

$$
\boldsymbol{A}_{c i j} \boldsymbol{R}=\left(\boldsymbol{A}_{i}-\boldsymbol{B}_{i} \boldsymbol{K}_{j} \boldsymbol{C}\right) \boldsymbol{R},
$$

then, since $r=m$, it is possible to eliminate the bi-linearity in (34) by setting

$$
B_{i} K_{j} C R=B_{i} K_{j} M M^{-1} C R=B_{i} N_{j} C,
$$

where

$$
\boldsymbol{K}_{j} \boldsymbol{M}=\boldsymbol{N}_{j}, \quad \boldsymbol{M}^{-1} \boldsymbol{C}=\boldsymbol{C R}^{-1}
$$

and $\boldsymbol{M} \in \mathbb{R}^{m \times m}$ is a regular square matrix. This implies (12) as well as (15), since

$$
\boldsymbol{A}_{c i j} \boldsymbol{R}=\boldsymbol{A}_{i} \boldsymbol{R}-\boldsymbol{B}_{i} \boldsymbol{K}_{j} \boldsymbol{C}=\boldsymbol{H}_{i j} .
$$

Using the membership functions properties, it can write for (19), (20),

$$
\boldsymbol{q}^{T}(t) \boldsymbol{Z}(\boldsymbol{\theta}(t)) \boldsymbol{q}(t)=\left[\begin{array}{c}
\mathrm{h}_{1}(\boldsymbol{\theta}(t) \boldsymbol{P} \boldsymbol{q}(t) \\
\mathrm{h}_{2}(\boldsymbol{\theta}(t) \boldsymbol{P} \boldsymbol{q}(t) \\
\vdots \\
\mathrm{h}_{s}(\boldsymbol{\theta}(t) \boldsymbol{P} \boldsymbol{q}(t)
\end{array}\right]^{T} \boldsymbol{R}^{\bullet} \boldsymbol{X}^{\bullet} \boldsymbol{R}^{\bullet}\left[\begin{array}{c}
\mathrm{h}_{1}(\boldsymbol{\theta}(t) \boldsymbol{P} \boldsymbol{q}(t) \\
\mathrm{h}_{2}(\boldsymbol{\theta}(t) \boldsymbol{P} \boldsymbol{q}(t) \\
\vdots \\
\mathrm{h}_{s}(\boldsymbol{\theta}(t) \boldsymbol{P} \boldsymbol{q}(t)
\end{array}\right]>0
$$




$$
\boldsymbol{q}^{T}(t) \boldsymbol{Z}(\boldsymbol{\theta}(t)) \boldsymbol{q}(t)=\left[\begin{array}{c}
\mathrm{h}_{1}\left(\boldsymbol{\theta}(t) \boldsymbol{q}^{\diamond}(t)\right. \\
\mathrm{h}_{2}\left(\boldsymbol{\theta}(t) \boldsymbol{q}^{\diamond}(t)\right. \\
\vdots \\
\mathrm{h}_{s}\left(\boldsymbol{\theta}(t) \boldsymbol{q}^{\diamond}(t)\right.
\end{array}\right]^{T} \boldsymbol{Y}^{\bullet}\left[\begin{array}{c}
\mathrm{h}_{1}\left(\boldsymbol{\theta}(t) \boldsymbol{q}^{\diamond}(t)\right. \\
\mathrm{h}_{2}\left(\boldsymbol{\theta}(t) \boldsymbol{q}^{\diamond}(t)\right. \\
\vdots \\
\mathrm{h}_{s}\left(\boldsymbol{\theta}(t) \boldsymbol{q}^{\diamond}(t)\right.
\end{array}\right]>0
$$

respectively, where

$$
\boldsymbol{q}^{\diamond}(t)=\boldsymbol{P} \boldsymbol{q}(t), \quad \boldsymbol{R}^{\bullet}=\operatorname{diag}\left[\begin{array}{llll}
\boldsymbol{R} & \boldsymbol{R} & \cdots & \boldsymbol{R}
\end{array}\right], \quad \boldsymbol{Y}^{\bullet}=\boldsymbol{R}^{\bullet} \boldsymbol{X}^{\bullet} \boldsymbol{R}^{\bullet}>0 .
$$

Subsequently, using the notations

$$
\boldsymbol{Y}_{i j}=\boldsymbol{R} \boldsymbol{X}_{i j} \boldsymbol{R},
$$

(32), (33) imply (13), (14), respectively, and (40) makes positiveness of (10). This concludes the proof.

Consider the case $r=m$ (square plants), where with each output signal is associated a reference signal. Such regime is called the forced regime and in this case is defined as follows:

Definition 4 A forced regime for the TS fuzzy system (1), (2) with the TS fuzzy static output controller (6) is foisted by the control policy

$$
\boldsymbol{u}_{c}(t)=-\sum_{j=1}^{s} h_{j}(\boldsymbol{\theta}(t)) \boldsymbol{K}_{j} \boldsymbol{C} \boldsymbol{q}(t)+\sum_{i=1}^{s} \sum_{j=1}^{s} h_{i}(\boldsymbol{\theta}(t)) h_{j}(\boldsymbol{\theta}(t)) \boldsymbol{W}_{i j} \boldsymbol{w}(t)
$$

where $\boldsymbol{w}(t) \in \mathbb{R}^{m}$ is desired output signal vector, and $\boldsymbol{W}_{i j} \in \mathbb{R}^{m \times m}, i, j=1,2, \ldots s$, is the set of signal gain matrices.

Theorem 7 If a square TS fuzzy system (1), (2) is stabilizable by the control policy (42) and for all $i$ are satisfied the rank conditions

$$
\operatorname{rank}\left[\begin{array}{cc}
\boldsymbol{A}_{i} & \boldsymbol{B}_{i} \\
\boldsymbol{C} & 0
\end{array}\right]=n+m
$$

then the matrices $\boldsymbol{W}_{i j}, i, j=1,2, \ldots$, s are given as

$$
\boldsymbol{W}_{i j}=-\left(\boldsymbol{C}\left(\boldsymbol{A}_{i}-\boldsymbol{B}_{i} \boldsymbol{K}_{j} \boldsymbol{C}\right)^{-1} \boldsymbol{B}_{i}\right)^{-1}
$$

and the relationship $\boldsymbol{y}_{s}=\boldsymbol{w}_{s}$ is achieved at a steady state of the closed-loop system, where $\boldsymbol{y}_{s}, \boldsymbol{w}_{s}$ are steady-state values of the vectors $\boldsymbol{y}(t), \boldsymbol{w}(t)$. 
Proof If all pairs $\left(\boldsymbol{A}_{i}, \boldsymbol{B}_{i}\right)$ are stabilizable and $\operatorname{rank} \boldsymbol{C}=\operatorname{rank} \boldsymbol{B}_{i}=m$ for all $i$, there exist such matrices $\boldsymbol{K}_{j}$ that $\boldsymbol{A}_{c i j}=\boldsymbol{A}_{i}-\boldsymbol{B}_{i} \boldsymbol{K}_{j} \boldsymbol{C}$ are stable matrices for all $i, j$ and, as consequence, $\operatorname{rank}\left(\boldsymbol{A}_{i}-\boldsymbol{B}_{i} \boldsymbol{K}_{j} \boldsymbol{C}\right)=n$. Because

$$
\operatorname{rank}\left[\begin{array}{cc}
\boldsymbol{I}_{n} & \mathbf{0} \\
-\boldsymbol{K}_{j} \boldsymbol{C} & \boldsymbol{I}_{m}
\end{array}\right]=n+m,
$$

then it yields for a $\boldsymbol{K}_{j}$ that

$$
\begin{gathered}
\operatorname{rank}\left[\begin{array}{cc}
\boldsymbol{A}_{i} & \boldsymbol{B}_{i} \\
\boldsymbol{C} & \mathbf{0}
\end{array}\right]= \\
=\operatorname{rank}\left[\begin{array}{cc}
\boldsymbol{A}_{i} & \boldsymbol{B}_{i} \\
\boldsymbol{C} & \mathbf{0}
\end{array}\right]\left[\begin{array}{cc}
\boldsymbol{I}_{n} & \mathbf{0} \\
-\boldsymbol{K}_{j} \boldsymbol{C} & \boldsymbol{I}_{m}
\end{array}\right]=\operatorname{rank}\left[\begin{array}{cc}
\boldsymbol{A}_{i}-\boldsymbol{B}_{i} \boldsymbol{K}_{j} \boldsymbol{C} & \boldsymbol{B}_{i} \\
\boldsymbol{C} & \mathbf{0}
\end{array}\right],
\end{gathered}
$$

while

$$
\begin{gathered}
\operatorname{rank}\left[\begin{array}{cc}
\boldsymbol{A}_{i}-\boldsymbol{B}_{i} \boldsymbol{K}_{j} \boldsymbol{C} & \boldsymbol{B}_{i} \\
\boldsymbol{C} & \mathbf{0}
\end{array}\right]= \\
=\operatorname{rank}\left[\begin{array}{cc}
\boldsymbol{I}_{n} & \mathbf{0} \\
-\boldsymbol{C}\left(\boldsymbol{A}_{i}-\boldsymbol{B}_{i} \boldsymbol{K}_{j} \boldsymbol{C}\right)^{-1} & \boldsymbol{I}_{m}
\end{array}\right]\left[\begin{array}{cc}
\boldsymbol{A}_{i}-\boldsymbol{B}_{i} \boldsymbol{K}_{j} \boldsymbol{C} & \boldsymbol{B}_{i} \\
\boldsymbol{C} & \mathbf{0}
\end{array}\right]= \\
=\operatorname{rank}\left[\begin{array}{cc}
\boldsymbol{A}_{i}-\boldsymbol{B}_{i} \boldsymbol{K}_{j} \boldsymbol{C} & \boldsymbol{B}_{i} \\
\mathbf{0} & -\boldsymbol{C}\left(\boldsymbol{A}_{i}-\boldsymbol{B}_{i} \boldsymbol{K}_{j} \boldsymbol{C}\right)^{-1} \boldsymbol{B}_{i}
\end{array}\right] .
\end{gathered}
$$

Because $\operatorname{rank}\left(\boldsymbol{A}_{i}-\boldsymbol{B}_{i} \boldsymbol{K}_{j} \boldsymbol{C}\right)=n$ and $\operatorname{rank} \boldsymbol{B}_{i}=m$, it has to be

$$
\operatorname{rank}\left(\boldsymbol{C}\left(\boldsymbol{A}_{i}-\boldsymbol{B}_{i} \boldsymbol{K}_{j} \boldsymbol{C}\right)^{-1} \boldsymbol{B}_{i}\right)=m,
$$

which implies (43).

In a steady-state, the disturbance-free equations (1), (2) and the control law equation (42) imply

$$
\begin{gathered}
\mathbf{0}=\sum_{i=1}^{s} \sum_{j=1}^{s} \mathrm{~h}_{i}\left(\boldsymbol{\theta}_{o}\right) \mathrm{h}_{j}\left(\boldsymbol{\theta}_{o}\right)\left(\left(\boldsymbol{A}_{i}-\boldsymbol{B}_{i} \boldsymbol{K}_{j} \boldsymbol{C}\right) \boldsymbol{q}_{s}+\boldsymbol{B}_{i} \boldsymbol{W}_{i j} \boldsymbol{w}_{s}\right), \\
\boldsymbol{y}_{s}=\boldsymbol{C} \boldsymbol{q}_{s},
\end{gathered}
$$

where $\boldsymbol{q}_{s}, \boldsymbol{y}_{s}, \boldsymbol{w}_{s}, \boldsymbol{\theta}_{o}$ are steady-state values of the vectors $\boldsymbol{q}(t), \boldsymbol{y}(t), \boldsymbol{w}(t), \boldsymbol{\theta}(t)$, respectively. Then for all $i, j$ it has to be satisfied

$$
\boldsymbol{q}_{s}=-\left(\boldsymbol{A}_{i}-\boldsymbol{B}_{i} \boldsymbol{K}_{j} \boldsymbol{C}\right)^{-1} \boldsymbol{B}_{i} \boldsymbol{W}_{i j} \boldsymbol{w}_{s}
$$

and the membership function property

$$
\sum_{i=1}^{s} \sum_{j=1}^{s} \mathrm{~h}_{i}(\boldsymbol{\theta}(t)) \mathrm{h}_{j}(\boldsymbol{\theta}(t))=1
$$


allows that (51) can be written as

$$
\begin{gathered}
\boldsymbol{q}_{s}=\sum_{i=1}^{s} \sum_{j=1}^{s} \mathrm{~h}_{i}(\boldsymbol{\theta}(t)) \mathrm{h}_{j}(\boldsymbol{\theta}(t)) \boldsymbol{q}_{s}= \\
=-\sum_{i=1}^{s} \sum_{j=1}^{s} \mathrm{~h}_{i}(\boldsymbol{\theta}(t)) \mathrm{h}_{j}(\boldsymbol{\theta}(t))\left(\boldsymbol{A}_{i}-\boldsymbol{B}_{i} \boldsymbol{K}_{j} \boldsymbol{C}\right)^{-1} \boldsymbol{B}_{i} \boldsymbol{W}_{i j} \boldsymbol{w}_{s} .
\end{gathered}
$$

Note, the condition (52) is satisfied at every time instant, and therefore in a steady state, too.

Since, according to (50), it is

$$
\boldsymbol{y}_{s}=-\sum_{i=1}^{s} \sum_{j=1}^{s} \mathrm{~h}_{i}(\boldsymbol{\theta}(t)) \mathrm{h}_{j}(\boldsymbol{\theta}(t)) \boldsymbol{C}\left(\boldsymbol{A}_{i}-\boldsymbol{B}_{i} \boldsymbol{K}_{j} \boldsymbol{C}\right)^{-1} \boldsymbol{B}_{i} \boldsymbol{W}_{i j} \boldsymbol{w}_{s}
$$

then, considering (9) and $\boldsymbol{y}_{s}=\boldsymbol{w}_{s}$, (54) implies (44). This concludes the proof.

It is evident that the static gains realized by the $\boldsymbol{G}_{i j}$ matrices are ideal in control if the plant parameters, on which the values of $\boldsymbol{W}_{i j}$ depend, are known and do not vary with time.

Note, the forced regime is basically designed for constant references and is very closely related to shift of origin.

\section{TS fuzzy virtual actuator}

In a faulty case with a single actuator fault the control structure is modified by adding the associated TS fuzzy virtual actuator block that masks the actuator fault, and allows the TS fuzzy controller to perceive the system as it was before the fault, i.e., the nominal TS fuzzy controller may still be used without it being necessary readjusted. To obtain the TS fuzzy virtual actuator state-space description, the following theorem is proven at first.

Theorem 8 (separation principle) The dynamics of TS fuzzy virtual actuator for the TS fuzzy system with a single actuator fault (3), (4) is given as

$$
\dot{\boldsymbol{e}}_{f a}(t)=\sum_{i=1}^{s} \sum_{j=1}^{s} h_{i}(\boldsymbol{\theta}(t)) h_{j}(\boldsymbol{\theta}(t))\left(\boldsymbol{A}_{c f i j} \boldsymbol{e}_{f a}(t)-\boldsymbol{B}_{i} \boldsymbol{u}_{c}(t)\right),
$$

where

$$
\begin{gathered}
\boldsymbol{A}_{c f i j}=\boldsymbol{A}_{i}-\boldsymbol{B}_{f i} \boldsymbol{G}_{j}, \\
\boldsymbol{e}_{f a}(t)=\boldsymbol{q}_{f a}(t)-\boldsymbol{q}(t)
\end{gathered}
$$

and $\boldsymbol{e}_{a}(t) \in \mathbb{R}^{n}, \boldsymbol{A}_{c f i j} \in \mathbb{R}^{n \times n}, i, j=1,2, \ldots s$. 
Proof Writing (1) and (4) compactly as

$$
\begin{gathered}
{\left[\begin{array}{c}
\dot{\boldsymbol{q}}_{f a}(t) \\
\dot{\boldsymbol{q}}(t)
\end{array}\right]=\sum_{i=1}^{s} \mathrm{~h}_{i}(\boldsymbol{\theta}(t))\left[\begin{array}{cc}
\boldsymbol{A}_{i} & \mathbf{0} \\
\mathbf{0} & \boldsymbol{A}_{i}
\end{array}\right]\left[\begin{array}{c}
\boldsymbol{q}_{f a}(t) \\
\boldsymbol{q}(t)
\end{array}\right]+} \\
+\sum_{i=1}^{s} \mathrm{~h}_{i}(\boldsymbol{\theta}(t))\left(\left[\begin{array}{cc}
\boldsymbol{B}_{f i} & \mathbf{0} \\
\mathbf{0} & \boldsymbol{B}_{i}
\end{array}\right]\left[\begin{array}{c}
\boldsymbol{u}_{f a}(t) \\
\boldsymbol{u}_{c}(t)
\end{array}\right]+\left[\begin{array}{c}
\boldsymbol{V}_{i} \\
\boldsymbol{V}_{i}
\end{array}\right] \boldsymbol{d}(t)\right),
\end{gathered}
$$

behavior of this extended system can be fully described also by using $\boldsymbol{q}_{f a}(t)$ and by the equation for the error vector $\boldsymbol{e}_{f a}(t)$ (57). Then, to perform the coordinate change, the transform matrix $\boldsymbol{T}$ can be defined with respect to (57) as follows

$$
\boldsymbol{T}=\left[\begin{array}{rr}
\boldsymbol{I} & 0 \\
\boldsymbol{I} & -\boldsymbol{I}
\end{array}\right], \quad \boldsymbol{T}^{-1}=\left[\begin{array}{rr}
\boldsymbol{I} & \mathbf{0} \\
\boldsymbol{I} & -\boldsymbol{I}
\end{array}\right],
$$

and, accordingly, it can be obtained

$$
\begin{gathered}
\boldsymbol{T}\left[\begin{array}{c}
\boldsymbol{q}_{f a}(t) \\
\boldsymbol{q}(t)
\end{array}\right]=\left[\begin{array}{rr}
\boldsymbol{I} & \mathbf{0} \\
\boldsymbol{I} & -\boldsymbol{I}
\end{array}\right]\left[\begin{array}{c}
\boldsymbol{q}_{f a}(t) \\
\boldsymbol{q}(t)
\end{array}\right]=\left[\begin{array}{l}
\boldsymbol{q}_{f a}(t) \\
\boldsymbol{e}_{f a}(t)
\end{array}\right], \\
{\left[\begin{array}{rr}
\boldsymbol{I} & \mathbf{0} \\
\boldsymbol{I} & -\boldsymbol{I}
\end{array}\right]\left[\begin{array}{c}
\boldsymbol{V}_{i} \\
\boldsymbol{V}_{i}
\end{array}\right]=\left[\begin{array}{c}
\boldsymbol{V}_{i} \\
\mathbf{0}
\end{array}\right], \quad\left[\begin{array}{rr}
\boldsymbol{I} & \mathbf{0} \\
\boldsymbol{I} & -\boldsymbol{I}
\end{array}\right]\left[\begin{array}{cc}
\boldsymbol{B}_{f i} & \mathbf{0} \\
\mathbf{0} & \boldsymbol{B}_{i}
\end{array}\right]=\left[\begin{array}{cc}
\boldsymbol{B}_{f i} & \mathbf{0} \\
\boldsymbol{B}_{f i} & -\boldsymbol{B}_{i}
\end{array}\right],} \\
{\left[\begin{array}{rr}
\boldsymbol{I} & \mathbf{0} \\
\boldsymbol{I} & -\boldsymbol{I}
\end{array}\right]\left[\begin{array}{rr}
\boldsymbol{A}_{i} & \mathbf{0} \\
\mathbf{0} & \boldsymbol{A}_{i}
\end{array}\right]\left[\begin{array}{rr}
\boldsymbol{I} & \mathbf{0} \\
\boldsymbol{I} & -\boldsymbol{I}
\end{array}\right]=\left[\begin{array}{cc}
\boldsymbol{A}_{i} & \mathbf{0} \\
\mathbf{0} & \boldsymbol{A}_{i}
\end{array}\right] .}
\end{gathered}
$$

Thus, (58) can be rewritten in the following form

$$
\begin{gathered}
{\left[\begin{array}{c}
\dot{\boldsymbol{q}}_{f a}(t) \\
\dot{\boldsymbol{q}}(t)
\end{array}\right]=\sum_{i=1}^{s} \mathrm{~h}_{i}(\boldsymbol{\theta}(t))\left[\begin{array}{cc}
\boldsymbol{A}_{i} & \mathbf{0} \\
\mathbf{0} & \boldsymbol{A}_{i}
\end{array}\right]\left[\begin{array}{c}
\boldsymbol{q}_{f a}(t) \\
\boldsymbol{q}(t)
\end{array}\right]+} \\
+\sum_{i=1}^{s} \mathrm{~h}_{i}(\boldsymbol{\theta}(t))\left(\left[\begin{array}{cc}
\boldsymbol{B}_{f i} & \mathbf{0} \\
\boldsymbol{B}_{f i} & -\boldsymbol{B}_{i}
\end{array}\right]\left[\begin{array}{c}
\boldsymbol{u}_{f a}(t) \\
\boldsymbol{u}_{c}(t)
\end{array}\right]+\left[\begin{array}{c}
\boldsymbol{V}_{i} \\
\mathbf{0}
\end{array}\right] \boldsymbol{d}(t)\right) .
\end{gathered}
$$

Defining the covering of the faulty control input as follows

$$
\boldsymbol{u}_{f a}(t)=-\sum_{j=1}^{s} \mathrm{~h}_{j}(\boldsymbol{\theta}(t)) \boldsymbol{G}_{j} \boldsymbol{e}_{f a}(t),
$$

where $\boldsymbol{G}_{j} \in \mathbb{R}^{r_{f} \times n}$, then the substitution of (64) in (63) leads to

$$
\begin{aligned}
& {\left[\begin{array}{c}
\dot{\boldsymbol{q}}_{f a}(t) \\
\dot{\boldsymbol{e}}_{f a}(t)
\end{array}\right]=\sum_{i=1}^{s} \mathrm{~h}_{i}(\boldsymbol{\theta}(t))\left(-\left[\begin{array}{c}
\mathbf{0} \\
\boldsymbol{B}_{i}
\end{array}\right] \boldsymbol{u}_{c}(t)+\left[\begin{array}{c}
\boldsymbol{V}_{i} \\
\mathbf{0}
\end{array}\right] \boldsymbol{d}(t)\right)+} \\
& +\sum_{i=1}^{s} \sum_{j=1}^{s} \mathrm{~h}_{i}(\boldsymbol{\theta}(t)) \mathrm{h}_{j}(\boldsymbol{\theta}(t))\left[\begin{array}{cc}
\boldsymbol{A}_{i} & -\boldsymbol{B}_{f i} \boldsymbol{G}_{j} \\
\mathbf{0} & \boldsymbol{A}_{i}-\boldsymbol{B}_{f i} \boldsymbol{G}_{j}
\end{array}\right]\left[\begin{array}{c}
\boldsymbol{q}_{f a}(t) \\
\boldsymbol{e}_{f a}(t)
\end{array}\right] .
\end{aligned}
$$


Thus, the second row of the equation (65) imply (55). This concludes the proof.

Obviously, in view of the block structure of the extended system matrix of the system (64), the separation principle holds and the gain matrices $\boldsymbol{G}_{j}$ can be designed independently on the TS fuzzy faulty system description if the couples $\left(\boldsymbol{A}_{i}, \boldsymbol{B}_{f i}\right)$ are controllable.

Corollary 3 The state-space description of the TS fuzzy faulty closed-loop system with activated TS fuzzy virtual actuator is as follows

$$
\begin{gathered}
\dot{\boldsymbol{q}}_{f a}(t)=\sum_{i=1}^{s} \sum_{j=1}^{s} h_{i}(\boldsymbol{\theta}(t)) h_{j}(\boldsymbol{\theta}(t))\left(\boldsymbol{A}_{c f i j} \boldsymbol{q}_{f a}(t)+\boldsymbol{V}_{f i} \boldsymbol{d}_{f a}(t)\right), \\
\boldsymbol{y}_{f a}(t)=\boldsymbol{C} \boldsymbol{q}_{f a}(t),
\end{gathered}
$$

where the structures of $\boldsymbol{V}_{f i} \in \mathbb{R}^{n \times r_{f a}}, \boldsymbol{d}_{f a} \in \mathbb{R}^{r_{f a}}, r_{f a}=r_{f}+r_{v}$, are

$$
\boldsymbol{V}_{f i}=\left[\begin{array}{ll}
\boldsymbol{B}_{f i} & \boldsymbol{V}_{i}
\end{array}\right], \quad \boldsymbol{d}_{f a}^{T}(t)=\left[\begin{array}{ll}
\boldsymbol{q}^{T}(t) \sum_{j=1}^{s} h_{j}(\boldsymbol{\theta}(t)) \boldsymbol{G}_{j}^{T} & \boldsymbol{d}^{T}(t)
\end{array}\right] .
$$

Thus, the TS fuzzy faulty system with an actuator fault, under control of TS fuzzy nominal static output controller covered by the TS fuzzy virtual actuator, operates in the reconfiguration regime along with the unknown input disturbance $\boldsymbol{d}_{f a}(t)$.

Proof Since the first row of the equation (65) gives

$$
\begin{aligned}
& \dot{\boldsymbol{q}}_{f a}(t)=\sum_{i=1}^{s} \sum_{j=1}^{s} \mathrm{~h}_{i}(\boldsymbol{\theta}(t)) \mathrm{h}_{j}(\boldsymbol{\theta}(t))\left(\boldsymbol{A}_{i} \boldsymbol{q}_{f a}(t)-\boldsymbol{B}_{f i} \boldsymbol{G}_{j} \boldsymbol{e}_{f a}(t)+\boldsymbol{V}_{i} \boldsymbol{d}(t)\right)= \\
& =\sum_{i=1}^{s} \sum_{j=1}^{s} \mathrm{~h}_{i}(\boldsymbol{\theta}(t)) \mathrm{h}_{j}(\boldsymbol{\theta}(t))\left(\boldsymbol{A}_{i} \boldsymbol{q}_{f a}(t)-\boldsymbol{B}_{f i} \boldsymbol{G}_{j}\left(\boldsymbol{q}_{f a}(t)-\boldsymbol{q}(t)\right)+\boldsymbol{V}_{i} \boldsymbol{d}(t)\right),
\end{aligned}
$$

then, using the notations (56), (68), the equation (69) implies (66) and (5) gives (56). This concludes the proof.

Corollary 4 The common description of TS fuzzy virtual actuator block is as follows

$$
\begin{gathered}
\dot{\boldsymbol{e}}_{f a}(t)=\sum_{i=1}^{s} \sum_{j=1}^{s} h_{i}(\boldsymbol{\theta}(t)) h_{j}(\boldsymbol{\theta}(t))\left(\boldsymbol{A}_{c f i j} \boldsymbol{e}_{f a}(t)-\boldsymbol{B}_{i} \boldsymbol{u}_{c}(t)\right), \\
\left.\boldsymbol{u}_{c}(t)=-\sum_{j=1}^{s} h_{j} \boldsymbol{\theta}(t)\right) \boldsymbol{K}_{j}\left(\boldsymbol{y}_{f a}(t)-\boldsymbol{C} \boldsymbol{e}_{f a}(t)\right) .
\end{gathered}
$$

Thus, in the autonomous regime, stability of TS fuzzy virtual actuator is determined by the same system matrix (56) as stability of the TS fuzzy closed-loop system in the reconfiguration regime.

If $\boldsymbol{u}_{c}(t)$ will be bounded also $\boldsymbol{e}_{f a}(t)$ will be bounded for any bounded $\boldsymbol{u}_{c}(t)$, which implies that

$$
\dot{\boldsymbol{e}}_{f a}(t)=\sum_{i=1}^{s} \sum_{j=1}^{s} h_{i}(\boldsymbol{\theta}(t)) h_{j}(\boldsymbol{\theta}(t)) \boldsymbol{A}_{c f i j} \boldsymbol{e}_{f a}(t)
$$

will be bounded-input bounded-output (BIBO) stable [6]. 
Proof Since it can write with (67)

$$
\boldsymbol{y}(t)=\boldsymbol{C} \boldsymbol{q}(t)=\boldsymbol{C}\left(\boldsymbol{q}_{f a}(t)-\left(\boldsymbol{q}_{f a}(t)-\boldsymbol{q}(t)\right)\right)=\boldsymbol{y}_{f a}(t)-\boldsymbol{C} \boldsymbol{e}_{f a}(t),
$$

substituting (73) into (6) gives (71). This concludes the proof.

\section{Design of TS fuzzy virtual actuators}

If all pairs $\left(\boldsymbol{A}_{i}, \boldsymbol{B}_{f i}\right)$ are controllable then within the given structure of TS fuzzy virtual actuators (70), the form of the unknown input disturbance $\boldsymbol{d}_{f a}(t)$ (68) and the system matrix (56), the conditions for design of TS fuzzy virtual actuator are given by the following theorem.

Theorem 9 TS fuzzy virtual actuator (70) is stable with quadratic constraint $\gamma^{\circ}$ if there exist a positive definite symmetric matrix $\boldsymbol{R}^{\circ} \in \mathbb{R}^{n \times n}$, matrices $\boldsymbol{Y}_{i j}^{\circ} \in \mathbb{R}^{n \times n}, \boldsymbol{N}_{j}^{\circ} \in \mathbb{R}^{r_{f} \times n}$ and a positive scalar $\gamma^{\circ} \in \mathbb{R}$ such that for all $i \in\langle 1,2, \ldots s\rangle, i<j \leqslant s, i, j \in\langle 1,2, \ldots s\rangle$, respectively, and $h_{i}(\boldsymbol{\theta}(t)) h_{j}(\boldsymbol{\theta}(t)) \neq 0$

$$
\begin{aligned}
& \boldsymbol{Y}^{\diamond}=\left[\begin{array}{cccc}
\boldsymbol{Y}_{11}^{\circ} & \boldsymbol{Y}_{12}^{\circ} & \cdots & \boldsymbol{Y}_{1 s}^{\circ} \\
\boldsymbol{Y}_{21}^{\circ} & \boldsymbol{Y}_{22}^{\circ} & \cdots & \boldsymbol{Y}_{2 s}^{\circ} \\
\vdots & \vdots & \ddots & \vdots \\
\boldsymbol{Y}_{s 1}^{\circ} & \boldsymbol{Y}_{s 2}^{\circ} & \cdots & \boldsymbol{Y}_{s s}^{\circ}
\end{array}\right]>0, \quad \boldsymbol{Y}_{i j}^{\circ}=\boldsymbol{Y}_{j i}^{\circ T}, \\
& \boldsymbol{R}^{\circ}=\boldsymbol{R}^{\circ T}>0, \\
& {\left[\begin{array}{ccc}
\boldsymbol{H}_{i i}^{\circ}+\boldsymbol{H}_{i i}^{\circ T}+\boldsymbol{Y}_{i i}^{\circ} & * & * \\
\boldsymbol{V}_{f i}^{T} & -\boldsymbol{\gamma} \boldsymbol{I}_{f a} & * \\
\boldsymbol{C R}^{\circ} & \mathbf{0} & -\boldsymbol{I}_{m}
\end{array}\right]<0,} \\
& {\left[\begin{array}{ccc}
\frac{\boldsymbol{H}_{i j}^{\circ}+\boldsymbol{H}_{j i}^{\circ}}{2}+\frac{\boldsymbol{H}_{i j}^{\circ}+\boldsymbol{H}_{j i}^{\circ T}}{2}+\frac{\boldsymbol{Y}_{i j}^{\circ}+\boldsymbol{Y}_{j i}^{\circ}}{2} & * & * \\
\frac{\boldsymbol{V}_{f i}^{T}+\boldsymbol{V}_{f j}^{T}}{2} & -\gamma \boldsymbol{I}_{r a} & * \\
\boldsymbol{C} \boldsymbol{R}^{\circ} & \mathbf{0} & -\boldsymbol{I}_{m}
\end{array}\right]<0,}
\end{aligned}
$$

where

$$
\boldsymbol{H}_{i j}^{\circ}=\boldsymbol{A}_{i} \boldsymbol{R}^{\circ}-\boldsymbol{B}_{f i} \boldsymbol{N}_{j}^{\circ} .
$$

Then, if the above conditions hold, the gain matrices $\boldsymbol{G}_{j}$ of the TS fuzzy virtual actuator associated with a single actuator fault are computed as

$$
\boldsymbol{G}_{j}=\boldsymbol{N}_{j}^{\circ}\left(\boldsymbol{R}^{\circ}\right)^{-1}, j=1,2, \ldots, s .
$$


Proof Defining the Lyapunov function as follows

$$
v\left(\boldsymbol{q}_{f a}(t)\right)=\boldsymbol{q}_{f a}^{T}(t) \boldsymbol{P}^{\circ} \boldsymbol{q}_{f a}(t)+\int_{0}^{t}\left(\boldsymbol{y}_{f a}^{T}(r) \boldsymbol{y}_{f a}(r)-\gamma^{\circ} \boldsymbol{d}_{f a}^{T}(r) \boldsymbol{d}_{f a}(r)\right) \mathrm{d} r>0,
$$

where $\boldsymbol{P}^{\circ}=\boldsymbol{P}^{\circ T}>0, \boldsymbol{P}^{\circ} \in \mathbb{R}^{n \times n}$, and $\boldsymbol{\gamma}^{\circ}>0$ is square of the $\mathrm{H}_{\infty}$ norm of the enhanced disturbance transfer functions matrix, then, evaluating the time derivative of $v\left(\boldsymbol{q}_{f a}(t)\right)$, it yields

$$
\dot{v}\left(\boldsymbol{q}_{f a}(t)\right)=\dot{\boldsymbol{q}}_{f a}^{T}(t) \boldsymbol{P}^{\circ} \boldsymbol{q}_{f a}(t)+\boldsymbol{q}_{f a}^{T}(t) \boldsymbol{P}^{\circ} \dot{\boldsymbol{q}}_{f a}(t)+\boldsymbol{y}_{f a}^{T}(t) \boldsymbol{y}_{f a}(t)-\boldsymbol{\gamma}^{\circ} \boldsymbol{d}_{f a}^{T}(t) \boldsymbol{d}_{f a}(t)<0 .
$$

Substituting (66), (67) into (81), and introducing the term

$$
\begin{gathered}
v_{f a}(\boldsymbol{\theta}(t))=\boldsymbol{q}_{f a}^{T}(t) \mathbf{Z}^{\circ}(\boldsymbol{\theta}(t)) \boldsymbol{q}_{f a}(t), \\
\mathbf{Z}^{\circ}(\boldsymbol{\theta}(t))=\sum_{i=1}^{s} \sum_{j=1}^{s} \mathrm{~h}_{i}(\boldsymbol{\theta}(t)) \mathrm{h}_{j}(\boldsymbol{\theta}(t)) \boldsymbol{X}_{i j}^{\circ}>0,
\end{gathered}
$$

while $\left\{\boldsymbol{X}_{i j}^{\circ}=\boldsymbol{X}_{i j}^{\circ T} \in \mathbb{R}^{n \times n}, i, j=1,2, \ldots, s\right\}$, then it can be set up

$$
\begin{gathered}
\dot{v}\left(\boldsymbol{q}_{f a}(t)\right)= \\
=\sum_{i=1}^{s} \sum_{j=1}^{s} \mathrm{~h}_{i}(\boldsymbol{\theta}(t)) \mathrm{h}_{j}(\boldsymbol{\theta}(t)) \boldsymbol{q}_{f a}^{T}(t)\left(\boldsymbol{P}^{\circ} \boldsymbol{A}_{c f i j}+\boldsymbol{A}_{c f i j}^{T} \boldsymbol{P}^{\circ}\right) \boldsymbol{q}_{f a}(t)+ \\
+\boldsymbol{q}_{f a}^{T}(t) \boldsymbol{P}^{\circ} \boldsymbol{V}_{f i} \boldsymbol{d}_{f a}(t)+\boldsymbol{d}_{f a}^{T}(t) \boldsymbol{V}_{f i}^{T} \boldsymbol{P}^{\circ} \boldsymbol{d}_{f a}(t)+ \\
+\boldsymbol{q}_{f a}^{T}(t) \boldsymbol{C}^{T} \boldsymbol{C} \boldsymbol{q}_{f a}(t)-\boldsymbol{\gamma}^{\circ} \boldsymbol{d}_{f a}^{T}(t) \boldsymbol{d}_{f a}(t) \leqslant \\
\leqslant-\sum_{i=1}^{s} \sum_{j=1}^{s} \mathrm{~h}_{i}(\boldsymbol{\theta}(t)) \mathrm{h}_{j}(\boldsymbol{\theta}(t)) \boldsymbol{q}_{f a}^{T}(t) \boldsymbol{X}_{i j}^{\circ} \boldsymbol{q}_{f a}(t)<0 .
\end{gathered}
$$

Using the notation

$$
\boldsymbol{q}_{f a}^{\circ T}(t)=\left[\begin{array}{ll}
\boldsymbol{q}_{f a}^{T}(t) & \boldsymbol{d}_{f a}^{T}(t)
\end{array}\right]
$$

(84) can be rewritten as

$$
\dot{v}\left(\boldsymbol{q}_{f a}^{\circ}(t)\right)=\sum_{i=1}^{s} \sum_{j=1}^{s} \mathrm{~h}_{i}(\boldsymbol{\theta}(t)) \mathrm{h}_{j}(\boldsymbol{\theta}(t)) \boldsymbol{q}_{f a}^{\circ T}(t) \boldsymbol{P}_{c i j}^{\circ} \boldsymbol{q}_{f a}^{\circ}(t)<0,
$$

where

$$
\boldsymbol{P}_{c i j}^{\circ}=\left[\begin{array}{cc}
\boldsymbol{P}^{\circ} \boldsymbol{A}_{c f i j}+\boldsymbol{A}_{c f i j}^{T} \boldsymbol{P}^{\circ}+\boldsymbol{X}_{i j}^{\circ}+\boldsymbol{C}^{T} \boldsymbol{C} & \boldsymbol{P}^{\circ} \boldsymbol{V}_{f i} \\
\boldsymbol{V}_{f i}^{T} \boldsymbol{P}^{\circ} & -\boldsymbol{\gamma}^{\circ} \boldsymbol{I}_{r_{f a}}
\end{array}\right]<0 .
$$

Permuting the subscripts $i$ and $j$ with respect to (86), and adding the result to (86), it yields

$$
2 \dot{v}\left(\boldsymbol{q}_{f a}^{\circ}(t)\right)==\sum_{i=1}^{s} \sum_{j=1}^{s} \mathrm{~h}_{i}(\boldsymbol{\theta}(t)) \mathrm{h}_{j}(\boldsymbol{\theta}(t)) \boldsymbol{q}_{f a}^{\circ T}(t)\left(\boldsymbol{P}_{c i j}^{\circ}+\boldsymbol{P}_{c j i}^{\circ}\right) \boldsymbol{q}_{f a}^{\circ}(t)<0
$$


and, by rearranging, (88) takes the form

$$
\begin{gathered}
\dot{v}\left(\boldsymbol{q}_{f a}^{\circ}(t)\right)=\sum_{i=1}^{s} \mathrm{~h}_{i}^{2}(\boldsymbol{\theta}(t)) \boldsymbol{q}_{f a}^{\circ T}(t) \boldsymbol{P}_{c i i}^{\circ} \boldsymbol{q}_{f a}^{\circ}(t)+ \\
+2 \sum_{i=1}^{s-1} \sum_{j=i+1}^{s} \mathrm{~h}_{i}(\boldsymbol{\theta}(t)) \mathrm{h}_{j}(\boldsymbol{\theta}(t)) \boldsymbol{q}_{f a}^{\circ T}(t) \frac{\boldsymbol{P}_{c i j}^{\circ}+\boldsymbol{P}_{c i i}^{\circ}}{2} \boldsymbol{q}_{f a}^{\circ}(t)<0 .
\end{gathered}
$$

Then, for all $i \in\langle 1,2, \ldots s\rangle, i<j \leqslant s, i, j \in\langle 1,2, \ldots s\rangle$, respectively,

$$
\boldsymbol{P}_{c i i}^{\circ}<0, \quad \frac{\boldsymbol{P}_{c i j}^{\circ}+\boldsymbol{P}_{c j i}^{\circ}}{2}<0 .
$$

Premultiplying the left-hand side and post-multiplying the right-hand side of the matrices $\boldsymbol{P}_{c j i}^{\circ}$ by the transform matrix

$$
\boldsymbol{S}^{\circ}=\operatorname{diag}\left[\begin{array}{ll}
\boldsymbol{R}^{\circ} & \boldsymbol{I}_{r_{f a}}
\end{array}\right], \quad \boldsymbol{R}^{\circ}=\left(\boldsymbol{P}^{\circ}\right)^{-1},
$$

(87), (90) give

$$
\begin{aligned}
& {\left[\begin{array}{cc}
\boldsymbol{A}_{c f i i} \boldsymbol{R}^{\circ}+\boldsymbol{R}^{\circ} \boldsymbol{A}_{c f i i}^{T}+\boldsymbol{R}^{\circ} \boldsymbol{X}_{i i}^{\circ} \boldsymbol{R}^{\circ}+\boldsymbol{R}^{\circ} \boldsymbol{C}^{T} \boldsymbol{C} \boldsymbol{R}^{\circ} & \boldsymbol{V}_{f i} \\
\boldsymbol{V}_{f i}^{T} & -\boldsymbol{\gamma}^{\circ} \boldsymbol{I}_{r f a}
\end{array}\right]<0,} \\
& {\left[\begin{array}{cc}
\frac{\boldsymbol{A}_{c f i j}+\boldsymbol{A}_{c f j i}}{2} \boldsymbol{R}^{\circ}+\boldsymbol{R}^{\circ} \frac{\boldsymbol{A}_{c f i j}^{T}+\boldsymbol{A}_{c f j i}^{T}+\boldsymbol{R}^{\circ} \frac{\boldsymbol{X}_{i j}^{\circ}+\boldsymbol{X}_{j i}^{\circ}}{2} \boldsymbol{R}^{\circ}+\boldsymbol{R}^{\circ} \boldsymbol{C}^{T} \boldsymbol{C} \boldsymbol{R}^{\circ}}{\frac{\boldsymbol{V}_{f i}+\boldsymbol{V}_{f i}}{2}} \\
\frac{\boldsymbol{V}_{f i}^{T}+\boldsymbol{V}_{f j}^{T}}{2} & -\boldsymbol{\gamma}^{\circ} \boldsymbol{I}_{r_{f a}}
\end{array}\right]<0,}
\end{aligned}
$$

which implies the equivalent inequalities

$$
\begin{aligned}
& {\left[\begin{array}{ccc}
\boldsymbol{A}_{c f i i} \boldsymbol{R}^{\circ}+\boldsymbol{R}^{\circ} \boldsymbol{A}_{c f i i}^{T}+\boldsymbol{R}^{\circ} \boldsymbol{X}_{i i}^{\circ} \boldsymbol{R}^{\circ} & \boldsymbol{V}_{f i} & \boldsymbol{R}^{\circ} \boldsymbol{C}^{T} \\
\boldsymbol{V}_{f i}^{T} & -\boldsymbol{\gamma}^{\circ} \boldsymbol{I}_{r_{f a}} & \mathbf{0} \\
\boldsymbol{C R}^{\circ} & \mathbf{0} & -\boldsymbol{I}_{m}
\end{array}\right]<0,} \\
& {\left[\begin{array}{ccc}
\frac{\boldsymbol{A}_{c f i j}+\boldsymbol{A}_{c f j i}}{2} \boldsymbol{R}^{\circ}+\boldsymbol{R}^{\circ} \frac{\boldsymbol{A}_{c f i j}^{T}+\boldsymbol{A}_{c f j i}^{T}}{2}+\boldsymbol{R}^{\circ} \frac{\boldsymbol{X}_{i j}^{\circ}+\boldsymbol{X}_{j i}^{\circ}}{2} \boldsymbol{R}^{\circ} & \frac{\boldsymbol{V}_{a i}+\boldsymbol{V}_{a i}}{2} & \boldsymbol{R}^{\circ} \boldsymbol{C}^{T} \\
\frac{\boldsymbol{V}_{a i}^{T}+\boldsymbol{V}_{a j}^{T}}{2} & -\boldsymbol{\gamma}^{\circ} \boldsymbol{I}_{f a} & \mathbf{0} \\
\boldsymbol{C} \boldsymbol{R}^{\circ} & \mathbf{0} & -\boldsymbol{I}_{m}
\end{array}\right]<0,}
\end{aligned}
$$

while

$$
\boldsymbol{A}_{c f i j} \boldsymbol{R}^{\circ}=\left(\boldsymbol{A}_{i}-\boldsymbol{B}_{f i} \boldsymbol{G}_{j}\right) \boldsymbol{R}^{\circ}=\boldsymbol{H}_{i j}^{\circ}, \quad \boldsymbol{N}_{j}^{\circ}=\boldsymbol{G}_{j} \boldsymbol{R}^{\circ} .
$$

Analogously to (38) it is

$$
\boldsymbol{q}_{f a}^{T}(t) \boldsymbol{Z}^{\circ}(\boldsymbol{\theta}(t)) \boldsymbol{q}_{f a}(t)=\left[\begin{array}{c}
\mathrm{h}_{1}\left(\boldsymbol{\theta}(t) \boldsymbol{P}^{\circ} \boldsymbol{q}_{f a}(t)\right. \\
\mathrm{h}_{2}\left(\boldsymbol{\theta}(t) \boldsymbol{P}^{\circ} \boldsymbol{q}_{f a}(t)\right. \\
\vdots \\
\mathrm{h}_{s}\left(\boldsymbol{\theta}(t) \boldsymbol{P}^{\circ} \boldsymbol{q}_{f a}(t)\right.
\end{array}\right]^{T} \boldsymbol{R}^{\diamond} \boldsymbol{X}^{\circ} \boldsymbol{R}^{\diamond}\left[\begin{array}{c}
\mathrm{h}_{1}\left(\boldsymbol{\theta}(t) \boldsymbol{P}^{\circ} \boldsymbol{q}_{f a}(t)\right. \\
\mathrm{h}_{2}\left(\boldsymbol{\theta}(t) \boldsymbol{P}^{\circ} \boldsymbol{q}_{f a}(t)\right. \\
\vdots \\
\mathrm{h}_{s}\left(\boldsymbol{\theta}(t) \boldsymbol{P}^{\circ} \boldsymbol{q}_{f a}(t)\right.
\end{array}\right]>0,
$$




$$
\boldsymbol{q}_{f a}^{T}(t) \boldsymbol{Z}^{\circ}(\boldsymbol{\theta}(t)) \boldsymbol{q}_{f a}(t)=\left[\begin{array}{c}
\mathrm{h}_{1}\left(\boldsymbol{\theta}(t) \boldsymbol{q}_{f a}^{\diamond}(t)\right. \\
\mathrm{h}_{2}\left(\boldsymbol{\theta}(t) \boldsymbol{q}_{f a}^{\diamond}(t)\right. \\
\vdots \\
\mathrm{h}_{s}\left(\boldsymbol{\theta}(t) \boldsymbol{q}_{f a}^{\diamond}(t)\right.
\end{array}\right]^{T} \boldsymbol{Y}^{\diamond}\left[\begin{array}{c}
\mathrm{h}_{1}\left(\boldsymbol{\theta}(t) \boldsymbol{q}_{f a}^{\diamond}(t)\right. \\
\mathrm{h}_{2}\left(\boldsymbol{\theta}(t) \boldsymbol{q}_{f a}^{\diamond}(t)\right. \\
\vdots \\
\mathrm{h}_{s}\left(\boldsymbol{\theta}(t) \boldsymbol{q}_{f a}^{\diamond}(t)\right.
\end{array}\right]>0
$$

respectively, where

$$
\boldsymbol{q}_{f a}^{\diamond}(t)=\boldsymbol{P}^{\circ} \boldsymbol{q}_{f a}(t), \boldsymbol{R}^{\diamond}=\operatorname{diag}\left[\begin{array}{llll}
\boldsymbol{R}^{\circ} & \boldsymbol{R}^{\circ} & \cdots & \boldsymbol{R}^{\circ}
\end{array}\right], \boldsymbol{Y}^{\diamond}=\boldsymbol{R}^{\diamond} \boldsymbol{X}^{\circ} \boldsymbol{R}^{\diamond}>0 .
$$

Subsequently, using the notations

$$
\boldsymbol{Y}_{i j}^{\circ}=\boldsymbol{R}^{\circ} \boldsymbol{X}_{i j}^{\circ} \boldsymbol{R}^{\circ}
$$

(94), (95) imply (76), (77), respectively, and (91) makes positiveness of (74). This concludes the proof.

The role of the forced regime for TS fuzzy controller is to force the faulty system in the steady-state the desired values of the reconfigurable output signals.

Theorem 10 A forced regime for the TS fuzzy system (1), (2) with the TS fuzzy static output controller (6) and activated virtual actuator is foisted by the control policy

$$
\boldsymbol{u}_{c}(t)=-\sum_{j=1}^{s} h_{j}(\boldsymbol{\theta}(t)) \boldsymbol{K}_{j} \boldsymbol{y}_{f a}(t)+\sum_{i=1}^{s} \sum_{j=1}^{s} h_{i}(\boldsymbol{\theta}(t)) h_{j}(\boldsymbol{\theta}(t))\left(\boldsymbol{W}_{i j}+\boldsymbol{W}_{i j}^{\circ}\right) \boldsymbol{w}(t),
$$

where

$$
\boldsymbol{W}_{i j}^{\circ}=\boldsymbol{C}\left(\boldsymbol{A}_{i}-\boldsymbol{B}_{f i} \boldsymbol{G}_{j}-\boldsymbol{B}_{i} \boldsymbol{K}_{j} \boldsymbol{C}\right)^{-1} \boldsymbol{B}_{i} \boldsymbol{K}_{j},
$$

$\boldsymbol{W}_{i j}$ is given in (44) and $\boldsymbol{w}(t) \in \mathbb{R}^{m}$ is desired output signal vector, and $\boldsymbol{W}_{i j}, \boldsymbol{W}_{i j}^{\circ} \in \mathbb{R}^{m \times m}$, $i, j=1,2, \ldots s$.

Proof Since (70), (71) implies

$$
\dot{\boldsymbol{e}}_{f a}(t)=\sum_{i=1}^{s} \sum_{j=1}^{s} \mathrm{~h}_{i}(\boldsymbol{\theta}(t)) \mathrm{h}_{j}(\boldsymbol{\theta}(t))\left(\left(\boldsymbol{A}_{c f i j}-\boldsymbol{B}_{i} \boldsymbol{K}_{j} \boldsymbol{C}\right) \boldsymbol{e}_{f a}(t)+\boldsymbol{B}_{i} \boldsymbol{K}_{j} \boldsymbol{y}_{f a}(t)\right),
$$

in a steady-state of the closed-loop with virtual actuator the equation (103) gives

$$
\mathbf{0}=\sum_{i=1}^{s} \sum_{j=1}^{s} \mathrm{~h}_{i}\left(\boldsymbol{\theta}_{o}\right) \mathrm{h}_{j}\left(\boldsymbol{\theta}_{o}\right)\left(\left(\boldsymbol{A}_{c f i j}-\boldsymbol{B}_{i} \boldsymbol{K}_{j} \boldsymbol{C}\right) \boldsymbol{e}_{f a o}+\boldsymbol{B}_{i} \boldsymbol{K}_{j} \boldsymbol{y}_{f a o}\right),
$$

where $\boldsymbol{e}_{f a o}, \boldsymbol{y}_{f a o}, \boldsymbol{\theta}_{o}$ are steady-state values of the vectors $\boldsymbol{e}_{f a}(t), \boldsymbol{y}_{f a}(t), \boldsymbol{\theta}(t)$, respectively. This means that it has to be satisfied for all $i, j$,

$$
\boldsymbol{e}_{f a o}=-\left(\boldsymbol{A}_{c f i j}-\boldsymbol{B}_{i} \boldsymbol{K}_{j} \boldsymbol{C}\right)^{-1} \boldsymbol{B}_{i} \boldsymbol{K}_{j} \boldsymbol{y}_{f a o} .
$$


Since it is desired that $\boldsymbol{y}_{f a o}=\boldsymbol{w}_{o}$, considering the membership function property (52) then (105) can be rewritten as

$$
\begin{gathered}
\boldsymbol{e}_{f a o}=\sum_{i=1}^{s} \sum_{j=1}^{s} \mathrm{~h}_{i}(\boldsymbol{\theta}(t)) \mathrm{h}_{j}(\boldsymbol{\theta}(t)) \boldsymbol{e}_{f a o}= \\
=-\sum_{i=1}^{s} \sum_{j=1}^{s} \mathrm{~h}_{i}(\boldsymbol{\theta}(t)) \mathrm{h}_{j}(\boldsymbol{\theta}(t))\left(\boldsymbol{A}_{c f i j}-\boldsymbol{B}_{i} \boldsymbol{K}_{j} \boldsymbol{C}\right)^{-1} \boldsymbol{B}_{i} \boldsymbol{K}_{j} \boldsymbol{w}_{o} .
\end{gathered}
$$

With respect to desired output variables at the steady-state of the closed-loop system with activated virtual actuator, it can write for the forced mode that

$$
\boldsymbol{e}_{f a}(t)=-\sum_{i=1}^{s} \sum_{j=1}^{s} \mathrm{~h}_{i}(\boldsymbol{\theta}(t)) \mathrm{h}_{j}(\boldsymbol{\theta}(t))\left(\boldsymbol{A}_{c f i j}-\boldsymbol{B}_{i} \boldsymbol{K}_{j} \boldsymbol{C}\right)^{-1} \boldsymbol{B}_{i} \boldsymbol{K}_{j} \boldsymbol{w}(t) .
$$

Then, substituting (73) into (42), it is obtained

$$
\boldsymbol{u}_{c}(t)=-\sum_{j=1}^{s} \mathrm{~h}_{j}(\boldsymbol{\theta}(t)) \boldsymbol{K}_{j}\left(\boldsymbol{y}_{f a}(t)-\boldsymbol{C} \boldsymbol{e}_{f a}(t)\right)+\sum_{i=1}^{s} \sum_{j=1}^{s} \mathrm{~h}_{i}(\boldsymbol{\theta}(t)) \mathrm{h}_{j}(\boldsymbol{\theta}(t)) \boldsymbol{W}_{i j} \boldsymbol{w}(t)
$$

and (107) together with (108) takes the form

$$
\begin{gathered}
\boldsymbol{u}_{c}(t)=-\sum_{j=1}^{s} \mathrm{~h}_{j}(\boldsymbol{\theta}(t)) \boldsymbol{K}_{j} \boldsymbol{C} \boldsymbol{y}_{f a}(t)+ \\
+\sum_{i=1}^{s} \sum_{j=1}^{s} \mathrm{~h}_{i}(\boldsymbol{\theta}(t)) \mathrm{h}_{j}(\boldsymbol{\theta}(t))\left(\boldsymbol{W}_{i j}+\boldsymbol{C}\left(\boldsymbol{A}_{c f i j}-\boldsymbol{B}_{i} \boldsymbol{K}_{j} \boldsymbol{C}\right)^{-1} \boldsymbol{B}_{i} \boldsymbol{K}_{j}\right) \boldsymbol{w}(t) .
\end{gathered}
$$

Thus, using the notations (56), (102), then (109) implies (101). This concludes the proof.

\section{Illustrative example}

The three-tank system with three input and three output variables is described by the set of equations

$$
\begin{gathered}
\frac{d q_{1}(t)}{d t}=\frac{u_{1}(t)}{F_{1}}-\frac{\alpha_{1} \operatorname{sign}\left[q_{1}(t)-q_{2}(t)\right] \sqrt{2 g\left|q_{1}(t)-q_{2}(t)\right|}}{F_{1} \sum_{i=1}^{3} \lambda_{i} q_{i}(t)} \lambda_{i} q_{i}(t), \\
\frac{d q_{2}(t)}{d t}=\frac{u_{2}(t)}{F_{2}}-\frac{\alpha_{2} \sqrt{2 g q_{2}(t)}}{F_{2} q_{2}(t)} q_{2}(t)+\frac{\alpha_{1} \operatorname{sign}\left[q_{1}(t)-q_{2}(t)\right] \sqrt{2 g\left|q_{1}(t)-q_{2}(t)\right|}}{F_{1} \sum_{i=1}^{3} \lambda_{i} q_{i}(t)} \sum_{i=1}^{3} \lambda_{i} q_{i}(t)+ \\
+\frac{\alpha_{3} \operatorname{sign}\left[x_{3}(t)-x_{2}(t)\right] \sqrt{2 g\left|x_{3}(t)-x_{2}(t)\right|} \sum_{i=1}^{3} \eta_{i} q_{i}(t)}{F_{3} \sum_{i=1}^{3} \eta_{i} x_{i}(t)}
\end{gathered}
$$




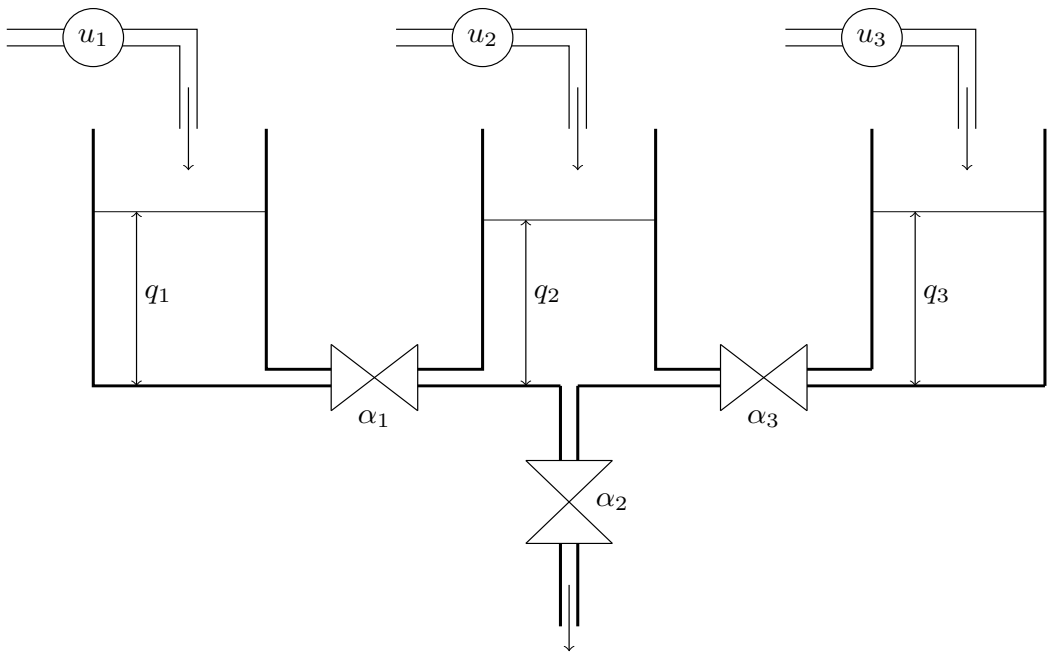

Figure 1. The three-tank system

$$
\begin{gathered}
\frac{d q_{3}(t)}{d t}=\frac{u_{3}(t)}{F_{3}}-\frac{\alpha_{3} \operatorname{sign}\left[q_{3}(t)-q_{2}(t)\right] \sqrt{2 g\left|q_{3}(t)-q_{2}(t)\right|}}{F_{3} \sum_{i=1}^{3} \eta_{i} q_{i}(t)} \eta_{i} q_{i}(t), \\
y_{k}(t)=q_{k}(t), k=1,2,3,
\end{gathered}
$$

where the measured output variables $y_{k}(t)$ are water levels $q_{k}(t), k=1,2,3$ in tanks [m] and the incoming flows are considered as the input variables $u_{k}(t), k=1,2,3\left[\mathrm{~m}^{3} / \mathrm{s}\right]$, while the bounds of the output and input variables were

$$
\begin{aligned}
& q_{1}^{\min }=q_{3}^{\min }=0.02[\mathrm{~m}], \quad q_{2}^{\min }=0.01[\mathrm{~m}], \\
& q_{1}^{\max }=q_{3}^{\max }=1.00[\mathrm{~m}], \quad q_{2}^{\max }=0.95[\mathrm{~m}], \\
& u_{1,2,3}^{\min }=0\left[\mathrm{~m}^{3} / \mathrm{s}\right], \quad u_{1,2,3}^{\max }=0.005\left[\mathrm{~m}^{3} / \mathrm{s}\right]
\end{aligned}
$$


The model parameters are

$$
\begin{aligned}
& g \text { - the gravitational acceleration } 9.80665\left(\mathrm{~m} / \mathrm{s}^{2}\right), \\
& F_{k} \text { - the same section of all tanks } \mathrm{k}=1,2,30.25\left(\mathrm{~m}^{2}\right), \\
& \alpha_{1} \text { - the equivalent section of the pipe } \\
& \quad \text { between the first and the second tank } 6.5 \times 10^{-4}\left(\mathrm{~m}^{2}\right), \\
& \alpha_{3} \text { - the equivalent section of the pipe } \\
& \quad \text { between the third and the second tank } 6.5 \times 10^{-4}\left(\mathrm{~m}^{2}\right) \text {, } \\
& \alpha_{2} \text { - the equivalent section of the outlet pipe } \\
& \quad \text { from the second tank } 9.0 \times 10^{-4}\left(\mathrm{~m}^{2}\right), \\
& \lambda_{k}, \eta_{k}-\text { real scalars, } \\
& \operatorname{sign}(\cdot)-\text { sign function. }
\end{aligned}
$$

Respecting the nonlinear structure of the system dynamic equations, and minimizing the number of premise variables, the premise variables were chosen as

$$
\begin{gathered}
\theta_{1}(t)=\frac{\alpha_{1} \operatorname{sign}\left[q_{1}(t)-q_{2}(t)\right] \sqrt{2 g\left|q_{1}(t)-q_{2}(t)\right|}}{F_{1} \sum_{i=1}^{3} \lambda_{i} x_{i}(t)}, \\
\theta_{2}(t)=\frac{\alpha_{2}}{F_{2}} \sqrt{\frac{2 g}{q_{2}(t)}}, \\
\theta_{3}(t)=\frac{\alpha_{3} \operatorname{sign}\left[q_{3}(t)-q_{2}(t)\right] \sqrt{2 g\left|q_{3}(t)-q_{2}(t)\right|}}{F_{3} \sum_{i=1}^{3} \eta_{i} q_{i}(t)},
\end{gathered}
$$

which, with the resulting sector bounds for premise variables, computed from the given bounds on input variables, and under the following numbering of combinations of the sector bounds

$$
\begin{array}{ll}
i=1 \leftarrow\left(\theta_{1}^{\text {max }}, \theta_{2}^{\text {max }}, \theta_{3}^{\text {max }}\right), & i=2 \leftarrow\left(\theta_{1}^{\text {max }}, \theta_{2}^{\text {max }}, \theta_{3}^{\text {min }}\right), \\
i=3 \leftarrow\left(\theta_{1}^{\text {max }}, \theta_{2}^{\text {min }}, \theta_{3}^{\text {max }}\right), & i=4 \leftarrow\left(\theta_{1}^{\text {max }}, \theta_{2}^{\text {min }}, \theta_{3}^{\text {min }}\right), \\
i=5 \leftarrow\left(\theta_{1}^{\text {min }}, \theta_{2}^{\text {max }}, \theta_{3}^{\text {max }}\right), & i=6 \leftarrow\left(\theta_{1}^{\text {min }}, \theta_{2}^{\text {max }}, \theta_{3}^{\text {min }}\right), \\
i=7 \leftarrow\left(\theta_{1}^{\text {min }}, \theta_{2}^{\text {min }}, \theta_{3}^{\text {max }}\right), & i=8 \leftarrow\left(\theta_{1}^{\text {min }}, \theta_{2}^{\text {min }}, \theta_{3}^{\text {min }}\right),
\end{array}
$$

imply the structures of the local sub-system matrices

$$
\boldsymbol{A}_{i}=\left[\begin{array}{rrr}
-\lambda_{1} \theta_{1}^{i} & -\lambda_{2} \theta_{1}^{i} & -\lambda_{3} \theta_{1}^{i} \\
\lambda_{1} \theta_{1}^{i}+\eta_{1} \theta_{3}^{i} & \lambda_{2} \theta_{1}^{i}+\eta_{2} \theta_{3}^{i}-\theta_{2}^{i} & \lambda_{3} \theta_{1}^{i}+\eta_{3} \theta_{3}^{i} \\
-\eta_{1} \theta_{3}^{i} & -\eta_{2} \theta_{3}^{i} & -\eta_{3} \theta_{3}^{i}
\end{array}\right]
$$




$$
\boldsymbol{B}=\left[\begin{array}{ccc}
\frac{1}{F_{1}} & 0 & 0 \\
0 & \frac{1}{F_{2}} & 0 \\
0 & 0 & \frac{1}{F_{3}}
\end{array}\right], \quad \boldsymbol{C}=\left[\begin{array}{ccc}
1 & 0 & 0 \\
0 & 1 & 0 \\
0 & 0 & 1
\end{array}\right]
$$

The choice of real scalars $\lambda_{k}, \eta_{k}, k=1,2,3$, gives the possibility to obtain different parameters of the matrix $\boldsymbol{A}_{i}$ (different linear parameter varying (LPV) form of the systems) [12]. The general limitation is that the couples $\left(\boldsymbol{A}_{i}, \boldsymbol{B}\right)$ have to be stabilizable and the couples $\left(\boldsymbol{A}_{i}, \boldsymbol{C}\right)$ have to be detectable for all $i$. In this sense the scalars were interactive setting as

$$
\lambda_{1}=0.20, \quad \lambda_{2}=0.69, \quad \lambda_{3}=0.16, \quad \eta_{1}=0.69, \quad \eta_{2}=-0.36, \quad \eta_{3}=0.06,
$$

which results in the following TS model matrix parameters

$$
\left.\begin{array}{l}
\boldsymbol{A}_{1}=\left[\begin{array}{rrr}
-0.0163 & -0.0563 & -0.0132 \\
0.1340 & -0.1832 & 0.0229 \\
-0.1178 & 0.0623 & -0.0097 \\
-0.0163 & -0.0563 & -0.0132 \\
0.1340 & -0.0242 & 0.0229 \\
-0.1178 & 0.0623 & -0.0097 \\
0.0033 & 0.0116 & 0.0027 \\
0.1145 & -0.2511 & 0.0070 \\
-0.1178 & 0.0623 & -0.0097 \\
0.0033 & 0.0116 & 0.0027 \\
0.1145 & -0.0921 & 0.0070 \\
-0.1178 & 0.0623 & -0.0097
\end{array}\right], \boldsymbol{A}_{3}=\left[\begin{array}{rrr}
-0.0163 & -0.0563 & -0.0132 \\
-0.0060 & -0.1091 & 0.0114 \\
0.0223 & -0.0118 & 0.0018 \\
-0.0163 & -0.0563 & -0.0132 \\
-0.0060 & 0.0499 & 0.0114 \\
0.0223 & -0.0118 & 0.0018 \\
0.0033 & 0.0116 & 0.0027 \\
-0.0256 & -0.1770 & -0.0046 \\
0.0223 & -0.0118 & 0.0018 \\
0.0033 & 0.0116 & 0.0027 \\
-0.0256 & -0.0180 & -0.0046 \\
0.0223 & -0.0118 & 0.0018
\end{array}\right], \\
\boldsymbol{A}_{5}=, \\
\boldsymbol{A}_{7}=\left[\begin{array}{rrr}
4 & 0 & 0 \\
0 & 4 & 0 \\
0 & 0 & 4
\end{array}\right], \quad \boldsymbol{A _ { 8 }}
\end{array}\right],
$$


The controller gain matrices $\boldsymbol{K}_{h}$ was synthesized by solving (10)-(14) using the SeDuMi package [16] while with $\gamma=1.7387$ the following controller gain matrices were produced

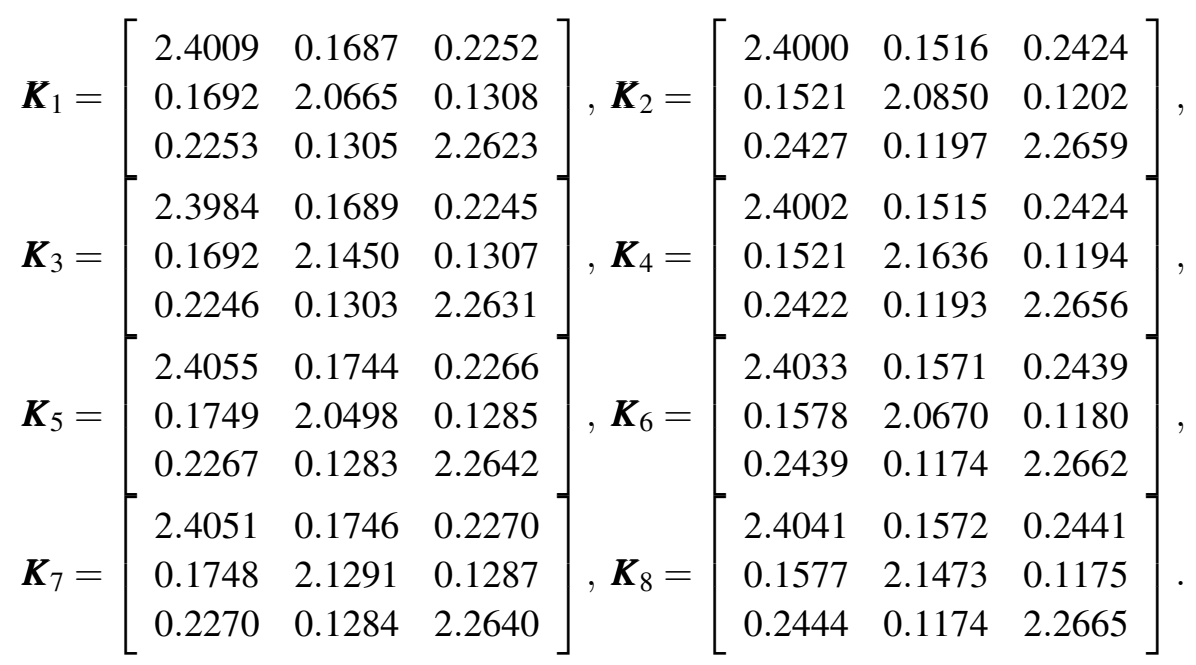

Note, the design condition results stable sets of the closed-loop subsystems matrix eigenvalues.

Considering the second actuator fault (the second column of $\boldsymbol{B}$ is zero column), then by solving (74)-(77) the gain matrices were designed for TS fuzzy virtual actuator as follows

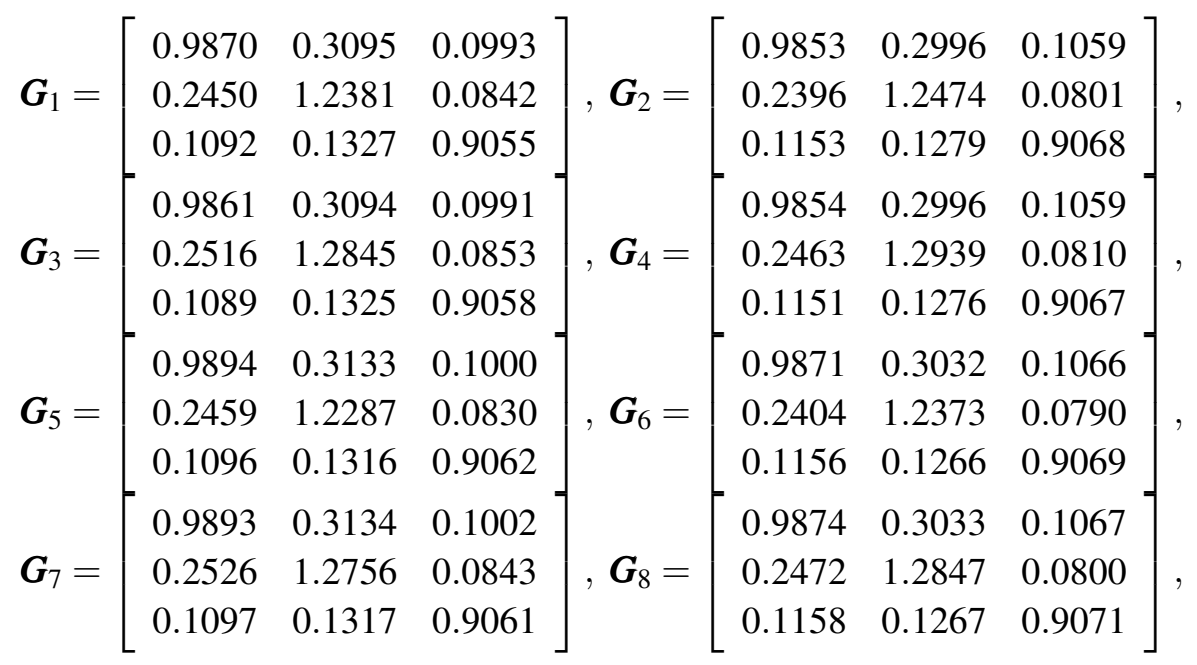

where $\gamma^{\circ}=5.7914$.

In the simulation, the forced mode was established for the TS fuzzy controller and the system initial conditions $\boldsymbol{q}^{T}(0)=\left[\boldsymbol{q}_{1}^{\min } \boldsymbol{q}_{2}^{\min } \boldsymbol{q}_{3}^{\min }\right]$ and $\boldsymbol{w}^{T}(t)=[0.650 .550 .60]$. As the results, Fig. 2 presents the system outputs response reflecting the second actuator fault. Starting and continuing the system activity in nominal conditions to the time instant $t=$ 


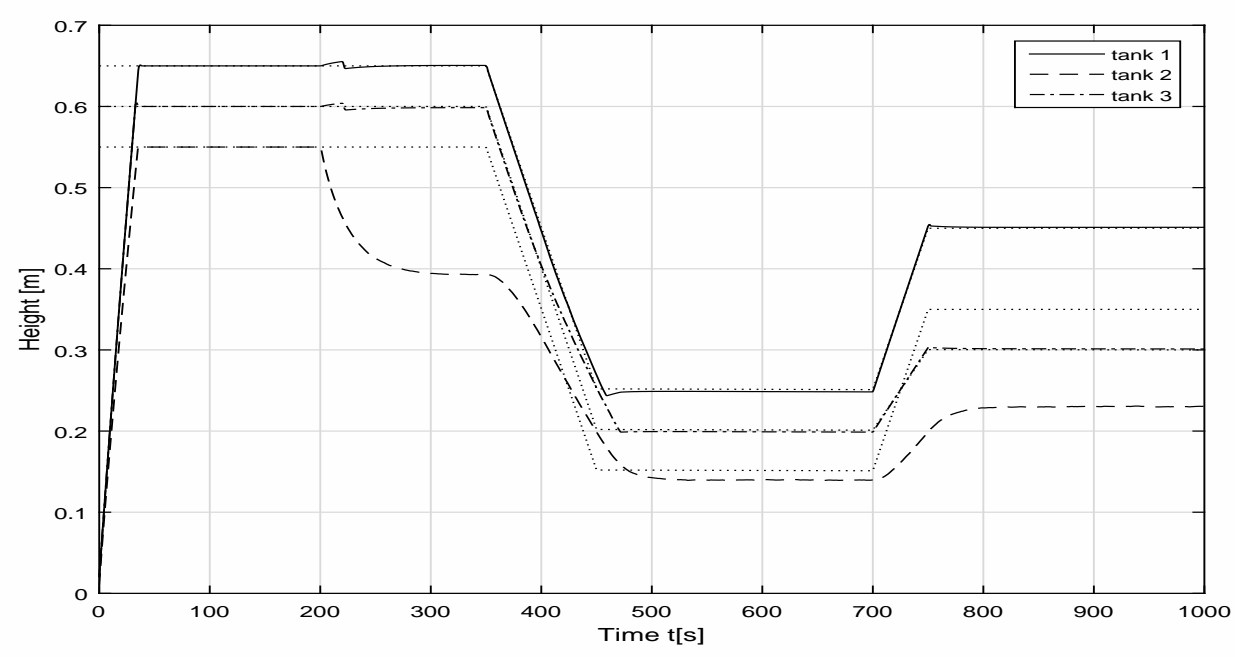

Figure 2. System output responses

$200 s$ when the second actuator fault was being occurred, the TS fuzzy virtual actuator in the forced mode was activated at the time instant $t=220 \mathrm{~s}$. Working further with activated TS fuzzy virtual actuator, the desired output was changed to $\boldsymbol{w}^{T}(t)=\left[\begin{array}{lll}0.25 & 0.15 & 0.20\end{array}\right]$ at the time instant $t=400 \mathrm{~s}$ and, subsequently, to $\boldsymbol{w}^{T}(t)=\left[\begin{array}{lll}0.45 & 0.35 & 0.30\end{array}\right]$ at the time instant $t=700 \mathrm{~s}$. It is clear, following the intervention of TS fuzzy virtual actuator in the operative area, the first and the third output of the system was stabilized at the desired levels. In the given structure of the system, it can not set the desired level value in the second tank in case of failure of the second actuator, but this level value is stabilized in the dependency on the desired level values of the first and third tanks.

\section{Concluding Remarks}

The proposed $\mathrm{H}_{\infty}$ based method gives new design features offered in collection of feasible algorithms for TS fuzzy virtual actuator design. The design conditions are accounted in terms of bounded real lemma structure of LMIs and exploit the standard numerical optimization. The TS fuzzy virtual actuator block is performed as an autonomous algorithm that may be activated with dependence on the fault detection and isolation subsystem response after singular actuator fault localization.

\section{References}

[1] H. Alwi, C. EdwardS and C.P. TAN: Fault Detection and Fault-Tolerant Control Using Sliding Modes, London, Springer, 2011. 
[2] A.M. Amani, A. Afshar and M.B. Menhaj: Fault tolerant networked control systems subject to actuator failure using virtual actuator technique. Prep. 18th IFAC World Congress, Milano, Italy, (2011), 5465-5470.

[3] M. Blanke, M. Kinnaert, J. Lunze and M. Staroswiecki: Diagnosis and Fault-Tolerant Control, Berlin, Springer-Verlag, 2006.

[4] M. Chadli and P. Borne: Multiple Models Approach in Automation. TakagiSugeno Fuzzy Systems, Hoboken, John Wiley \& Sons, 2013.

[5] C.A.R. CRusius and A. Trofino: Sufficient LMI conditions for output feedback control problems. IEEE Tran. Automatic Control, 44(5), (1999), 1053-1057.

[6] Z. GAO and P.J. ANTSAKLIS: Reconfigurable control system design via perfect model-following. In Proc. 1991 AIAA Guidance, Navigation and Control Conference, New Orleans, LA, USA, (1991), 239-246.

[7] W.M. Haddad and V. Chellaboina: Nonlinear Dynamical Systems and Control. A Lyapunov-Based Approach, Princeton, Princeton University Press, 2008.

[8] D. Krokavec and A. Filasová: Stabilizing fuzzy output control for a class of nonlinear systems. Advances in Fuzzy Systems, 2013(1), Article ID 294971, 9p, (2013).

[9] D. Krokavec, A. Filasová and P. LiščInskÝ: Dynamic output control of nonlinear systems described by Takagi-Sugeno models. Proc. 2014 IEEE Multiconf. on Systems and Control MSC 2014, Antibes, France, (2014), 959-964.

[10] D. Krokavec, A. Filasová and V. Serbák: FTC structures with virtual actuators and dynamic output controllers. Proc. 9th IFAC Symp. Fault Detection, Supervision and Safety for Technical Processes SAFEPROCESS'15, Paris, France, (2015), 511-516.

[11] D. Krokavec, A. Filasová and V. Serbák: FTC with dynamic virtual actuators: Characterization via dynamic output controllers and $\mathrm{H}_{\infty}$ approachs. Mathematical Problems in Engineering, 2016 Article ID 259056, 16p, (2015).

[12] A.M. Nagy, B. Marx, G. Mourot, G. Schutz and J. Ragot: State estimation of the three-tank system using a multiple model. Proc. Joint 48th IEEE Conf. on Decision and Control and 28th Chinese Control Conference, Shanghai, P.R. China, (2009), 7795-7800.

[13] J. LunZE and T. STEFFEN: Control reconfiguration after actuator failures using disturbance decoupling methods. IEEE Tran. Automatic Control, 51(10), (2006), 1590-1601. 
[14] M.S. Mahmoud and Y. XiA: Analysis and Synthesis of Fault-Tolerant Control Systems, Chichester, John Wiley \& Sons, 2014.

[15] H. Noura, D. Theilliol, J.C. Ponsart and A. Chamseddine: FaultTolerant Control Systems. Design and Practical Applications, London, Springer, 2009.

[16] D. Peaucelle, D. Henrion, Y Labit and K. Taitz: User's Guide for SeDuMi Interface 1.04, Toulouse, France: LAAS-CNRS, 2002.

[17] J.H. RichTER: Reconfigurable Control of Nonlinear Dynamical Systems. A FaultHiding Approach, Berlin, Springer-Verlag, 2011.

[18] T. STEFFEN: Control Reconfiguration of Dynamical Systems. Linear Approaches and Structural Tests, Berlin, Springer-Verlag, 2005.

[19] T. TAKAgi and M. SugEnO: Fuzzy identification of systems and its applications to modeling and control. IEEE Tran. Systems, Man, and Cybernetics, 15(1), (1985), 116-132.

[20] K. Tanaka and H.O. Wang: Fuzzy Control Systems Design and Analysis. A Linear Matrix Inequality Approach, New York, John Wiley \& Sons, 2001.

[21] M. Witczak: Fault Diagnosis and Fault-Tolerant Control Strategies for NonLinear Systems. Analytical and Soft Computing Approaches, Cham, Springer, 2014.

[22] H.O. WANG, K. TANAKA and M.F. GRIFFin: An approach to fuzzy control of nonlinear systems. Stability and design issues. IEEE Tran. Fuzzy Systems, 4(1), (1996), 14-23.

[23] K. ZHANG, B. JiANG and P. SHI: Observer-Based Fault Estimation and Accomodation for Dynamic Systems, Berlin, Springer-Verlag, 2013.

[24] A. Zolghadri, D. Henry, J. Cieslak, D. Efimov and P. Goupil: Fault Diagnosis and Fault-Tolerant Control and Guidance for Aerospace Vehicles. From Theory to Application, London, Springer, 2014. 